\title{
Existence of multiple positive solutions for fractional Laplace problems with critical growth and sign-changing weight in non-contractible domains
}

\section{Lu Pang ${ }^{1}$, Xueqin Li' and Yajing Zhang ${ }^{1 *}$ (I)}

"Correspondence:

zhangyj@sxu.edu.cn

${ }^{1}$ School of Mathematical Sciences,

Shanxi University, Taiyuan, China

\begin{abstract}
We prove the existence of multiple positive solutions for a fractional Laplace problem with critical growth and sign-changing weight in non-contractible domains.

MSC: 49J35; 35A15; 35S15

Keywords: Multiple positive solutions; Fractional Laplace problems; Critical growth; Lusternik-Schnirelmann category
\end{abstract}

\section{Introduction}

In this paper we consider the following critical problem involving fractional Laplacian:

$$
\begin{cases}(-\Delta)^{s} u=a(x) u^{p-1}+u^{2 *}-1 & \text { in } \Omega, \\ u>0 & \text { in } \Omega, \\ u=0 & \text { in } \mathbb{R}^{N} \backslash \Omega,\end{cases}
$$

where $s \in(0,1)$ is fixed and $(-\Delta)^{s}$ is the fractional Laplace operator, $\Omega \subset \mathbb{R}^{N}(N>2 s)$ is a smooth bounded domain, $1<p<2,2_{s}^{*}:=\frac{2 N}{N-2 s}$, and $a \in C(\bar{\Omega})$ changes sign in $\Omega$.

During the last years there has been an increasing interest in the study of the fractional Laplacian, motivated by great applications and by important advances in the theory of nonlinear partial differential equations, see $[3,7,11,14,15,17,20,21,24,25,35,36]$ for details. Nonlinear equations involving fractional Laplacian are currently actively studied. The fractional Laplace operator $(-\Delta)^{s}$ (up to normalization factors) may be defined as

$$
-(-\Delta)^{s} u(x)=\int_{\mathbb{R}^{N}}(u(x+y)+u(x-y)-2 u(x)) K(y) d y, \quad x \in \mathbb{R}^{N},
$$

where $K(x)=|x|^{-(N+2 s)}, x \in \mathbb{R}^{N}$. We will denote by $H^{s}\left(\mathbb{R}^{N}\right)$ the usual fractional Sobolev space endowed with the so-called Gagliardo norm

$$
\|u\|_{H^{s}\left(\mathbb{R}^{N}\right)}=\|u\|_{L^{2}\left(\mathbb{R}^{N}\right)}+\left(\int_{\mathbb{R}^{2 N}}|u(x)-u(y)|^{2} K(x-y) d x d y\right)^{1 / 2},
$$

(c) The Author(s) 2019. This article is distributed under the terms of the Creative Commons Attribution 4.0 International License (http://creativecommons.org/licenses/by/4.0/), which permits unrestricted use, distribution, and reproduction in any medium, provided you give appropriate credit to the original author(s) and the source, provide a link to the Creative Commons license, and indicate if changes were made. 
while $X_{0}$ is the function space defined as

$$
X_{0}=\left\{u \in H^{s}\left(\mathbb{R}^{N}\right): u=0 \text { a.e. in } \mathbb{R}^{N} \backslash \Omega\right\} .
$$

We refer to $[22,29,30]$ for a general definition of $X_{0}$ and its properties. The embedding $X_{0} \hookrightarrow L^{q}(\Omega)$ is continuous for any $q \in\left[1,2_{s}^{*}\right]$ and compact for any $q \in\left[1,2_{s}^{*}\right)$. The space $X_{0}$ is endowed with the norm defined as

$$
\|u\|_{X_{0}}=\left(\int_{\mathbb{R}^{2 N}}|u(x)-u(y)|^{2} K(x-y) d x d y\right)^{1 / 2} .
$$

By Lemma 5.1 in [29] we have $C_{0}^{2}(\Omega) \subset X_{0}$. Thus $X_{0}$ is nonempty. Note that $\left(X_{0},\|\cdot\|_{X_{0}}\right)$ is a Hilbert space with scalar product

$$
(u, v)_{X_{0}}=\int_{\mathbb{R}^{2 N}}(u(x)-u(y))(v(x)-v(y)) d x d y .
$$

It is well known that the following critical problem

$$
\begin{cases}-\Delta u=u^{2^{*}-1} & \text { in } \Omega, \\ u>0 & \text { in } \Omega, \\ u=0 & \text { on } \partial \Omega,\end{cases}
$$

has no positive solution if $\Omega$ is a star-shaped domain, where $2^{*}=\frac{2 N}{N-2}$. For a noncontractible domain $\Omega$, Coron [12] proved that (1.2) has a positive solution. Later, Bahri and Coron [4] improved Coron's existence result by showing, via topological arguments based upon homology theory, that (1.2) admits a positive solution provided that $H_{m}\left(\Omega, \mathbb{Z}_{2}\right) \neq\{0\}$ for some $m>0$. After that, many papers have studied the existence and multiplicity of positive solutions of the problem similar to $(1.2)$, see $[16,18,37,39]$.

It is natural to think that, as in the local case, by assuming suitable geometrical or topological conditions on $\Omega$, one can get the existence of nontrivial solutions for the nonlocal fractional problem. In a recent work, Secchi et al. [28] consider the following nonlocal fractional problem:

$$
\begin{cases}(-\Delta)^{s} u=u^{2_{s}^{*}-1} & \text { in } \Omega, \\ u>0 & \text { in } \Omega, \\ u=0 & \text { in } \mathbb{R}^{N} \backslash \Omega .\end{cases}
$$

They proved that (1.3) admits at least a positive solution if there is a point $x_{0} \in \mathbb{R}^{N}$ and radii $R_{2}>R_{1}>0$ such that

$$
\left\{R_{1} \leq\left|x-x_{0}\right| \leq R_{2}\right\} \subset \Omega, \quad\left\{\left|x-x_{0}\right| \leq R_{1}\right\} \not \subset \bar{\Omega}
$$

and $R_{2} / R_{1}$ is sufficiently large.

Motivated by the works mentioned above, we study problem (1.1), which involves the critical exponent, the effect of the coefficient $a(x)$, and the domain with "rich topology". 
We try to extend some important results, which are well known for the classical case of the Laplacian (see, e.g., Theorem 1.1 in [39]), to a nonlocal setting.

Taking into account that we are looking for positive solutions, we consider the energy functional associated with (1.1)

$$
\begin{aligned}
I(u)= & \frac{1}{2} \int_{\mathbb{R}^{2 N}}|u(x)-u(y)|^{2} K(x-y) d x d y \\
& -\frac{1}{p} \int_{\Omega} a(x)\left(u^{+}\right)^{p} d x-\frac{1}{2_{s}^{*}} \int_{\Omega}\left(u^{+}\right)^{2_{s}^{*}} d x,
\end{aligned}
$$

where $u^{+}=\max \{u, 0\}$ denotes the positive part of $u$. By the maximum principle (Proposition 2.2.8 in [33]), it is easy to check that critical points of $I$ are the positive solutions of (1.1).

We make the following assumptions:

(H1) There exist three constants $\rho_{2}>\rho_{1}>\rho_{0}>0$ such that $\bar{B}_{\rho_{2}}(0) \backslash B_{\rho_{1}}(0) \subset \Omega$ and $B_{\rho_{0}}(0) \cap \Omega=\emptyset$, where $B_{\rho}(0)=\left\{x \in \mathbb{R}^{N}:|x|<\rho\right\}$ for any $\rho>0$;

(H2) There exists a domain $\bar{B}_{\rho_{2}}(0) \backslash B_{\rho_{1}}(0) \subset \mathcal{D} \subset \Omega$ such that $a(x)>0$ for $x \in \mathcal{D}$ and $a(x) \leq 0$ for $x \in \Omega \backslash \mathcal{D}$.

Theorem 1.1 Assume that (H1), (H2) hold. Then there exists $\sigma_{0}>0$ such that if $\left|a^{+}\right|_{q}<\sigma_{0}$, where $a^{+}(x)=\max \{a(x), 0\}, q=\frac{2_{s}^{*}}{2_{s}^{*}-p}$, (1.1) has three positive solutions $\tilde{u}_{i}(1 \leq i \leq 3)$ such that

$$
\int_{\Omega} a(x) \tilde{u}_{i}^{q} d x>0, \quad i=1,2,3
$$

We should remark that $\tilde{u}_{2}$ and $\tilde{u}_{3}$ satisfy $I\left(\tilde{u}_{i}\right)<I\left(\tilde{u}_{1}\right)+\frac{s}{N} S_{s}^{\frac{N}{2 s}}(i=2,3)$, where $S_{s}$ is the Sobolev constant. It is an interesting task to find the fourth positive solution $\tilde{u}_{4}$ with $I\left(\tilde{u}_{4}\right)>$ $I\left(\tilde{u}_{1}\right)+\frac{s}{N} S_{s}^{\frac{N}{2 s}}$ provided $\rho_{2} / \rho_{1}$ is sufficiently large, although we shall not undertake it here.

This paper is organized as follows. In Sect. 2 we introduce Nehari manifold and state technical and elementary lemmas useful along the paper. In Sect. 3 we prove the existence of the first solution of (1.1). In Sect. 4 we establish some essential estimates of energy. In Sect. 5 we prove the existence of the other two solutions by Lusternik-Schnirelmann category. We denote by $|\cdot|_{r}$ the $L^{r}(\Omega)$-norm for any $r>1$, respectively.

\section{Preliminaries}

Recall that $I$ is unbounded from below; we can get rid of this problem once we restrict $I$ to the Nehari manifold

$$
\begin{aligned}
\mathcal{N} & =\left\{u \in X_{0} \backslash\{0\}:\left\langle I^{\prime}(u), u\right\rangle=0\right\} \\
& =\left\{u \in X_{0} \backslash\{0\}:\|u\|_{X_{0}}^{2}=\int_{\Omega} a(x)\left(u^{+}\right)^{p} d x+\int_{\Omega}\left(u^{+}\right)^{2_{s}^{*}} d x\right\} .
\end{aligned}
$$

Notice that $u^{+} \not \equiv 0$ for any $u \in \mathcal{N}$, and on $\mathcal{N}$ the functional $I$ reads

$$
I(u)=\left(\frac{1}{2}-\frac{1}{2_{s}^{*}}\right)\|u\|_{X_{0}}^{2}-\left(\frac{1}{p}-\frac{1}{2_{s}^{*}}\right) \int_{\Omega} a(x)\left(u^{+}\right)^{p} d x .
$$


Set

$$
q=\frac{2_{s}^{*}}{2_{s}^{*}-p} .
$$

In our context, the Sobolev constant is given by

$$
S_{s}=\inf _{u \in H^{s}\left(\mathbb{R}^{N}\right) \backslash\{0\}} \frac{\int_{\mathbb{R}^{2 N}}(u(x)-u(y))^{2} K(x-y) d x d y}{\left(\int_{\mathbb{R}^{N}}|u(x)|^{2} d x\right)^{2 / 2_{s}^{*}}} .
$$

Lemma 2.1 I is coercive and bounded from below on $\mathcal{N}$.

Proof If $u \in \mathcal{N}$, by (2.1) and the Sobolev inequality,

$$
I(u) \geq \frac{s}{N}\|u\|_{X_{0}}^{2}-\left(\frac{1}{p}-\frac{1}{2_{s}^{*}}\right)\left|a^{+}\right|_{q} S_{s}^{-p / 2}\|u\|_{X_{0}}^{p} .
$$

Since $1<p<2$, we get that $I$ is coercive and bounded from below on $\mathcal{N}$.

Define

$$
\psi(u)=\left\langle I^{\prime}(u), u\right\rangle
$$

Then, for $u \in \mathcal{N}$, we have

$$
\begin{aligned}
\left\langle\psi^{\prime}(u), u\right\rangle & =2\|u\|_{X_{0}}^{2}-p \int_{\Omega} a(x)\left(u^{+}\right)^{p} d x-2_{s}^{*} \int_{\Omega}\left(u^{+}\right)^{2_{s}^{*}} d x \\
& =(2-p)\|u\|_{X_{0}}^{2}-\left(2_{s}^{*}-p\right) \int_{\Omega}\left(u^{+}\right)^{2_{s}^{*}} d x \\
& =\left(2_{s}^{*}-p\right) \int_{\Omega} a(x)\left(u^{+}\right)^{p} d x-\left(2_{s}^{*}-2\right)\|u\|_{X_{0}}^{2} .
\end{aligned}
$$

Adopting a method similar to that used in [34], we split $\mathcal{N}$ into three parts:

$$
\begin{aligned}
& \mathcal{N}^{+}=\left\{u \in \mathcal{N}:\left\langle\psi^{\prime}(u), u\right\rangle>0\right\} ; \\
& \mathcal{N}^{0}=\left\{u \in \mathcal{N}:\left\langle\psi^{\prime}(u), u\right\rangle=0\right\} ; \\
& \mathcal{N}^{-}=\left\{u \in \mathcal{N}:\left\langle\psi^{\prime}(u), u\right\rangle<0\right\} .
\end{aligned}
$$

Lemma 2.2 Assume that $u$ is a minimizer for I on $\mathcal{N}$ and $u \notin \mathcal{N}^{0}$. Then $\left\langle I^{\prime}(u), v\right\rangle=0$ for any $v \in X_{0}$.

The proof is similar to that of Theorem 2.3 in [9], we omit it.

Set

$$
\sigma_{1}=\frac{2_{s}^{*}-2}{2_{s}^{*}-p}\left(\frac{2-p}{2_{s}^{*}-p}\right)^{(2-p) /\left(2_{s}^{*}-2\right)} S_{s}^{\left(2_{s}^{*}-p\right) /\left(2_{s}^{*}-2\right)} .
$$

Lemma 2.3 $\mathcal{N}^{0}=\emptyset$ if $\left|a^{+}\right|_{q}<\sigma_{1}$. 
Proof Assume by contradiction that there exists $a \in C(\bar{\Omega})$ with $\left|a^{+}\right|_{q}<\sigma_{1}$ such that $\mathcal{N} \neq \emptyset$. By (2.4) and (2.2), we have

$$
\|u\|_{X_{0}}^{2}=\frac{2_{s}^{*}-p}{2-p} \int_{\Omega}\left(u^{+}\right)^{2_{s}^{*}} d x \leq \frac{2_{s}^{*}-p}{2-p} S_{s}^{-2_{s}^{*} / 2}\|u\|_{X_{0}}^{2_{s}^{*}}
$$

Consequently,

$$
\|u\|_{X_{0}} \geq\left(\frac{2-p}{2_{s}^{*}-p} S_{s}^{2_{s}^{*} / 2}\right)^{1 /\left(2_{s}^{*}-2\right)} .
$$

Similarly, by (2.5), we have

$$
\|u\|_{X_{0}}^{2}=\frac{2_{s}^{*}-p}{2_{s}^{*}-2} \int_{\Omega} a(x)\left(u^{+}\right)^{p} d x \leq \frac{2_{s}^{*}-p}{2_{s}^{*}-2}\left|a^{+}\right|_{q} S_{s}^{-p / 2}\|u\|_{X_{0}}^{p},
$$

and so

$$
\|u\|_{X_{0}} \leq\left(\frac{2_{s}^{*}-p}{2_{s}^{*}-2}\left|a^{+}\right|_{q} S_{s}^{-p / 2}\right)^{\frac{1}{2-p}}
$$

Thus, we get that $\left|a^{+}\right|_{q} \geq \sigma_{1}$, which is impossible.

Define

$$
X_{0}^{+}:=\left\{u \in X_{0}: u^{+} \not \equiv 0\right\} .
$$

Lemma 2.4 For each $u \in X_{0}^{+}$, we have

(i) if $\int_{\Omega} a(x)\left(u^{+}\right)^{p} d x \leq 0$, then there exists unique $t^{-}(u)>t_{\max }$ such that $t^{-}(u) u \in \mathcal{N}^{-}$ and $\varphi(t):=I(t u)$ is increasing on $\left(0, t^{-}(u)\right)$ and decreasing on $\left(t^{-}(u),+\infty\right)$, where

$$
t_{\max }=\left(\frac{(2-p)\|u\|_{X_{0}}^{2}}{\left(2_{s}^{*}-p\right) \int_{\Omega}\left(u^{+}\right)^{2 *} d x}\right)^{\frac{N-2 s}{4 s}}
$$

Furthermore,

$$
\varphi\left(t^{-}(u)\right)=\sup _{t \geq 0} \varphi(t)
$$

(ii) If $\int_{\Omega} a(x)\left(u^{+}\right)^{p} d x>0$, then there exist unique $0<t^{+}(u)<t_{\max }<t^{-}(u)$ such that $t^{+}(u) u \in \mathcal{N}^{+}, t^{-}(u) u \in \mathcal{N}^{-}$, and $\varphi(t)$ is decreasing on $\left(0, t^{+}(u)\right) \cup\left(t^{-}(u),+\infty\right)$ and increasing on $\left(t^{+}(u), t^{-}(u)\right)$. Furthermore,

$$
\varphi\left(t^{+}(u)\right)=\inf _{0 \leq t \leq t^{-}(u)} \varphi(t), \quad \varphi\left(t^{-}(u)\right)=\sup _{t \geq t^{+}(u)} \varphi(t) .
$$

(iii) $t^{-}(u)$ is a continuous function for $u \in X_{0}^{+}$.

(iv) $\mathcal{N}^{-}=\left\{u \in X_{0}^{+}: \frac{1}{\|u\|_{X_{0}}} t^{-}\left(\frac{u}{\|u\|_{X_{0}}}\right)=1\right\}$. 
Proof Fix $u \in X_{0}^{+}$. We consider the following function:

$$
\gamma(t)=t^{2-p}\|u\|_{X_{0}}^{2}-t^{2_{s}^{*}-p} \int_{\Omega}\left(u^{+}\right)^{2_{s}^{*}} d x, \quad \forall t>0 .
$$

Clearly, $t u \in \mathcal{N}$ if and only if $\gamma(t)=\int_{\Omega} a(x)\left(u^{+}\right)^{p} d x$. Moreover,

$$
\gamma^{\prime}(t)=(2-p) t^{1-p}\|u\|_{X_{0}}^{2}-\left(2_{s}^{*}-p\right) t^{2_{s}^{*}-p-1} \int_{\Omega}\left(u^{+}\right)^{2_{s}^{*}} d x .
$$

So, it is easy to see that $t u \in \mathcal{N}^{+}$(or $\mathcal{N}^{-}$) if and only if $\gamma^{\prime}(t)>0$ (or $<0$ ). Notice that $\gamma$ is increasing on $\left(0, t_{\max }\right)$ and decreasing on $\left(t_{\max },+\infty\right)$ and $\gamma(t) \rightarrow-\infty$ as $t \rightarrow+\infty$.

(i) If $\int_{\Omega} a(x)\left(u^{+}\right)^{p} d x \leq 0$, then $\gamma(t)=\int_{\Omega} a(x)\left(u^{+}\right)^{p} d x$ has a unique solution $t^{-}(u)>t_{\max }$ and $\gamma^{\prime}\left(t^{-}(u)\right)<0$. Thus, $t^{-}(u) u \in \mathcal{N}^{-}$. Since

$$
\varphi^{\prime}(t)=t^{p-1}\left[\gamma(t)-\int_{\Omega} a(x)\left(u^{+}\right)^{p} d x\right],
$$

we get that (2.6) holds.

(ii) Assume that $\int_{\Omega} a(x)|u|^{p} d x>0$. Direct computation yields that

$$
\begin{aligned}
\gamma\left(t_{\max }\right) & =\left(\frac{(2-p)\|u\|_{X_{0}}^{2}}{\left(2_{s}^{*}-p\right) \int_{\Omega}|u|^{2_{s}^{*} d x}}\right)^{\frac{(N-2 s)(2-p)}{4 s}} \frac{2_{s}^{*}-2}{2_{s}^{*}-p}\|u\|_{X_{0}}^{2} \\
& \geq \frac{2_{s}^{*}-2}{2_{s}^{*}-p}\left(\frac{2-p}{2_{s}^{*}-p}\right)^{\frac{(N-2 s)(2-p)}{4 s}} S_{s}^{N(2-p) /(4 s)}\|u\|_{X_{0}}^{p} \\
& \geq \frac{2_{s}^{*}-2}{2_{s}^{*}-p}\left(\frac{2-p}{2_{s}^{*}-p}\right)^{\frac{(N-2 s)(2-p)}{4 s}} S_{s}^{\left(2_{s}^{*}-p\right) /\left(2_{s}^{*}-2\right)}\left|a^{+}\right|_{q}^{-1} \int_{\Omega} a(x)\left(u^{+}\right)^{p} d x \\
& >\int_{\Omega} a(x)\left(u^{+}\right)^{p} d x
\end{aligned}
$$

since $\left|a^{+}\right|_{q}<\sigma_{1}$. Thus, $\gamma(t)=\int_{\Omega} a(x)\left(u^{+}\right)^{p} d x$ has exactly two solutions $t^{+}(u)<t_{\max }<t^{-}(u)$ such that $\gamma^{\prime}\left(t^{+}(u)\right)>0$ and $\gamma^{\prime}\left(t^{-}(u)\right)<0$, and $\varphi(t)$ is decreasing on $\left(0, t^{+}(u)\right) \cup\left(t^{-}(u),+\infty\right)$ and increasing on $\left(t^{+}(u), t^{-}(u)\right)$. Consequently, $t^{+}(u) u \in \mathcal{N}^{+}$and $t^{-}(u) u \in \mathcal{N}^{-}$, and (2.7) holds.

(iii) The uniqueness of $t^{-}(u)$ and its extremal property give that $t^{-}(u)$ is a continuous function of $u$.

(iv) Set

$$
\mathcal{S}:=\left\{u \in X_{0}^{+}: \frac{1}{\|u\|_{X_{0}}} t^{-}\left(\frac{u}{\|u\|_{X_{0}}}\right)=1\right\} .
$$

Let $v=\frac{u}{\|u\|_{X_{0}}}$ for any $u \in \mathcal{N}^{-}$. By (i) and (ii), there exists $t^{-}(v)>0$ such that $t^{-}(v) v \in \mathcal{N}^{-}$, that is, $\frac{t^{-}(v)}{\|u\|_{X_{0}}} u \in \mathcal{N}^{-}$. Since $u \in \mathcal{N}^{-}$, we have $t^{-}(v)=\|u\|_{X_{0}}$. Hence, we get $\mathcal{N}^{-} \subset \mathcal{S}$. On the other hand, let $u \in \mathcal{S}$. Then,

$$
u=t^{-}\left(\frac{u}{\|u\|_{X_{0}}}\right) \frac{u}{\|u\|_{X_{0}}} \in \mathcal{N}^{-}
$$

Thus, $\mathcal{S} \subset \mathcal{N}^{-}$. 


\section{Existence of the first solution}

Define

$$
m^{+}=\inf _{u \in \mathcal{N}^{+}} I(u) \text { and } m^{-}=\inf _{u \in \mathcal{N}^{-}} I(u) .
$$

Set

$$
\sigma_{2}=\frac{p}{2} \sigma_{1}
$$

\section{Lemma 3.1}

(i) $m^{+}<0$ iffunction a satisfies $\left|a^{+}\right|_{q} \in\left(0, \sigma_{1}\right)$;

(ii) there exists positive constant $c_{0}$ such that $m^{-} \geq c_{0}$ if $\left|a^{+}\right|_{q}<\sigma_{2}$. In particular, $m^{+}=\inf _{u \in \mathcal{N}} I(u)$ if function a satisfies $\left|a^{+}\right|_{q} \in\left(0, \sigma_{2}\right)$.

Proof (i) If $u \in \mathcal{N}^{+}$, then by (2.5) we get that

$$
\|u\|_{X_{0}}^{2}<\frac{2_{s}^{*}-p}{2_{s}^{*}-2} \int_{\Omega} a(x)\left(u^{+}\right)^{p} d x .
$$

Thus, by (2.1),

$$
I(u)<-\left(1-\frac{p}{2_{s}^{*}}\right)\left(\frac{1}{p}-\frac{1}{2}\right) \int_{\Omega} a(x)\left(u^{+}\right)^{p} d x<0,
$$

and so $m^{+}<0$.

(ii) If $u \in \mathcal{N}^{-}$, then by (2.4),

$$
\frac{2-p}{2_{s}^{*}-p}\|u\|_{X_{0}}^{2}<\int_{\Omega}\left(u^{+}\right)^{2_{s}^{*}} d x \leq S_{s}^{-22_{s}^{*} / 2}\|u\|_{X_{0}}^{2_{s}^{*}} .
$$

Consequently,

$$
\|u\|_{X_{0}}>S_{s}^{N /(4 s)}\left(\frac{2-p}{2_{s}^{*}-p}\right)^{1 /\left(2_{s}^{*}-2\right)} .
$$

By (2.3) and $\left|a^{+}\right|_{q}<\sigma_{2}$, we have

$$
\begin{aligned}
I(u) \geq & \|u\|_{X_{0}}^{p}\left[\frac{s}{N}\|u\|_{X_{0}}^{2-p}-\left(\frac{1}{p}-\frac{1}{2_{s}^{*}}\right)\left|a^{+}\right|_{q} S_{s}^{-p / 2}\right] \\
\geq & S_{s}^{N p /(4 s)}\left(\frac{2-p}{2_{s}^{*}-p}\right)^{p /\left(2_{s}^{*}-2\right)}\left[\frac{s}{N} S_{s}^{N(2-p) /(4 s)}\left(\frac{2-p}{2_{s}^{*}-p}\right)^{(2-p) /\left(2_{s}^{*}-2\right)}\right. \\
& \left.-\left(\frac{1}{p}-\frac{1}{2_{s}^{*}}\right)\left|a^{+}\right|_{q} S_{s}^{-p / 2}\right] \\
> & 0 .
\end{aligned}
$$

From now on, we assume that $\left|a^{+}\right|_{q} \in\left(0, \sigma_{2}\right)$.

Lemma 3.2 I satisfies the $(P S)_{\beta}$ condition in $X_{0}$ for $\beta<m^{+}+\frac{s}{N} S_{s}^{\frac{N}{2 s}}$. 
Proof Let $\left\{u_{n}\right\}$ be a $(P S)_{\beta}$ sequence for $I$ such that

$$
I\left(u_{n}\right) \rightarrow \beta \quad \text { and } \quad I^{\prime}\left(u_{n}\right) \rightarrow 0
$$

Then, for $n$ big enough, we have

$$
\begin{aligned}
\beta+1+\left\|u_{n}\right\|_{X_{0}} & \geq I\left(u_{n}\right)-\frac{1}{2_{s}^{*}}\left\langle I^{\prime}\left(u_{n}\right), u_{n}\right\rangle \\
& =\left(\frac{1}{2}-\frac{1}{2_{s}^{*}}\right)\left\|u_{n}\right\|_{X_{0}}^{2}-\left(\frac{1}{p}-\frac{1}{2_{s}^{*}}\right) \int_{\Omega} a(x)\left(u_{n}^{+}\right)^{p} d x \\
& \geq\left(\frac{1}{2}-\frac{1}{2_{s}^{*}}\right)\left\|u_{n}\right\|_{X_{0}}^{2}-\left(\frac{1}{p}-\frac{1}{2_{s}^{*}}\right)\left|a^{+}\right|_{q} S_{s}^{-p / 2}\left\|u_{n}\right\|_{X_{0}}^{p} .
\end{aligned}
$$

It follows that $\left\|u_{n}\right\|_{X_{0}}$ is bounded. Going if necessary to a subsequence, we can assume that

$$
\begin{array}{ll}
u_{n} \rightarrow u_{0} & \text { in } X_{0}, \\
u_{n} \rightarrow u_{0} & \text { in } L^{r}(\Omega) \text { for } r \in\left[1,2_{s}^{*}\right), \\
u_{n} \rightarrow u_{0} & \text { a.e. in } \Omega .
\end{array}
$$

We derive from (3.1) that $\left\langle I^{\prime}\left(u_{0}\right), v\right\rangle=0, \forall v \in X_{0}$, i.e., $u_{0}$ is a solution of (1.1). In particular, $u_{0} \in \mathcal{N}$. Thus, by Lemma 3.1, we have $I\left(u_{0}\right) \geq m^{+}$. Since $X_{0}$ is a Hilbert space, we have

$$
\left\|u_{n}\right\|_{X_{0}}^{2}=\left\|u_{n}-u_{0}\right\|_{X_{0}}^{2}+\left\|u_{0}\right\|_{X_{0}}^{2}+o(1)
$$

By Brézis-Lieb’s lemma [8], we get

$$
\int_{\Omega}\left(u_{n}^{+}\right)^{2_{s}^{*}} d x=\int_{\Omega}\left(\left(u_{n}-u_{0}\right)^{+}\right)^{2_{s}^{*}} d x+\int_{\Omega}\left(u_{0}^{+}\right)^{2_{s}^{*}} d x+o(1) .
$$

Since $\left(u_{n}^{+}\right)^{2_{s}^{*}-1}$ is bounded in $L^{p^{\prime}}(\Omega)$ with $p^{\prime}=2_{s}^{*} /\left(2_{s}^{*}-1\right)$ and $L^{p^{\prime}}(\Omega)$ is a reflexible space, we get $\left(u_{n}^{+}\right)^{2_{s}^{*}-1} \rightarrow\left(u_{0}^{+}\right)^{2_{s}^{*}-1}$ in $L^{p^{\prime}}(\Omega)$, and so

$$
\int_{\Omega}\left(u_{n}^{+}\right)^{2_{s}^{*}-1} u_{0} d x \rightarrow \int_{\Omega}\left(u_{0}^{+}\right)^{2_{s}^{*}} d x
$$

Similarly, since $u_{n} \rightarrow u_{0}$ in $L^{2_{s}^{*}}(\Omega)$ and $\left(u_{0}^{+}\right)^{2_{s}^{*}-1} \in L^{p^{\prime}}(\Omega)$, we get

$$
\int_{\Omega}\left(u_{0}^{+}\right)^{2_{s}^{*}-1} u_{n} d x \rightarrow \int_{\Omega}\left(u_{0}^{+}\right)^{2_{s}^{*}} d x
$$

By (3.2)-(3.5), we have

$$
I\left(u_{n}\right)=I\left(u_{0}\right)+\frac{1}{2}\left\|u_{n}-u_{0}\right\|_{X_{0}}^{2}-\frac{1}{2_{s}^{*}} \int_{\Omega}\left(u^{+}\right)^{2_{s}^{*}} d x+o(1)
$$


and

$$
\begin{aligned}
o(1) & =\left\langle I^{\prime}\left(u_{n}\right)-I^{\prime}\left(u_{0}\right), u_{n}-u_{0}\right\rangle \\
& =\left\|u_{n}-u_{0}\right\|_{X_{0}}^{2}-\int_{\Omega}\left(\left(u_{n}-u_{0}\right)^{+}\right)^{2 *} d x+o(1) .
\end{aligned}
$$

By (3.6) and (3.7), we have

$$
\begin{aligned}
\frac{s}{N}\left\|u_{n}-u_{0}\right\|_{X_{0}}^{2} & =I\left(u_{n}\right)-I\left(u_{0}\right)+o(1) \\
& \leq I\left(u_{n}\right)-m^{+}+o(1) \\
& =\beta-m^{+}+o(1) .
\end{aligned}
$$

Thus, there exists a positive constant $\sigma>0$ such that

$$
\left\|u_{n}-u_{0}\right\|_{X_{0}}^{2}<S_{s}^{\frac{N}{2 s}}-\sigma
$$

for $n$ large enough. By (3.7), (3.8), and Sobolev inequality, we get

$$
\begin{aligned}
0 & <\left[1-\left(S_{s}^{\frac{N}{2 s}}-\sigma\right)^{\left(2_{s}^{*}-2\right) / 2} S_{s}^{-2_{s}^{*} / 2}\right]\left\|u_{n}-u_{0}\right\|_{X_{0}}^{2} \\
& \leq\left(1-S_{s}^{-2_{s}^{*} / 2}\left\|u_{n}-u_{0}\right\|_{X_{0}}^{2_{s}^{*}-2}\right)\left\|u_{n}-u_{0}\right\|_{X_{0}}^{2} \\
& \leq\left\|u_{n}-u_{0}\right\|_{X_{0}}^{2}-\int_{\Omega}\left(\left(u_{n}-u_{0}\right)^{+}\right)^{2_{s}^{*}} d x=o(1) .
\end{aligned}
$$

This implies $\left\|u_{n}-u_{0}\right\|_{X_{0}} \rightarrow 0$ in $X_{0}$.

Theorem 3.3 There exists a minimizer $\tilde{u}_{1}$ of the critical problem (1.1), and it satisfies

(i) $\tilde{u}_{1} \in \mathcal{N}^{+}$and $I\left(\tilde{u}_{1}\right)=m^{+}$;

(ii) $\tilde{u}_{1} \in C^{0, s}\left(\mathbb{R}^{N}\right)$ is a positive solution of $(1.1)$;

(iii) $I\left(\tilde{u}_{1}\right) \rightarrow 0$ as $\left|a^{+}\right|_{q} \rightarrow 0$.

Proof Applying Ekeland's variational principle [13] and using the similar argument as the proof of Theorem 1 in [34], we get that there exists $\left\{u_{n}\right\} \subset \mathcal{N}^{+}$such that

$$
I\left(u_{n}\right) \rightarrow m^{+} \quad \text { and } \quad I^{\prime}\left(u_{n}\right) \rightarrow 0 .
$$

By Lemma 3.2, there exist a subsequence (still denoted by $\left\{u_{n}\right\}$ ) and $\tilde{u}_{1} \in \mathcal{N}^{+}$, a solution of (1.1), such that $u_{n} \rightarrow \tilde{u}_{1}$ in $X_{0}$ and $m^{+}=I\left(\tilde{u}_{1}\right)$. By the maximum principle (Proposition 2.2.8 in [33]), $\tilde{u}_{1}$ is strictly positive in $\Omega$. By Proposition 2.2 in [6], $u \in L^{\infty}(\Omega)$. Furthermore, by Proposition 1.1 in [26] (or Proposition 5 in [31]), $u \in C^{0, s}\left(\mathbb{R}^{N}\right)$.

By (2.6),

$$
\left\|\tilde{u}_{1}\right\|_{X_{0}} \leq\left(\frac{2_{s}^{*}-p}{2_{s}^{*}-2}\left|a^{+}\right|_{q} S_{s}^{-p / 2}\right)^{\frac{1}{2-p}} .
$$

This implies $\left\|\tilde{u}_{1}\right\|_{X_{0}} \rightarrow 0$ as $\left|a^{+}\right|_{q} \rightarrow 0$, and so $I\left(\tilde{u}_{1}\right) \rightarrow 0$ as $\left|a^{+}\right|_{q} \rightarrow 0$. 


\section{Estimates of energy}

Recall that $S_{s}$ is defined as

$$
S_{s}:=\inf _{v \in H^{s}\left(\mathbb{R}^{N}\right) \backslash\{0\}} \frac{\int_{\mathbb{R}^{2 N}}|v(x)-v(y)|^{2} K(x-y) d x d y}{\left(\int_{\mathbb{R}^{N}}|v|^{2 *} d x\right)^{2 / 2_{s}^{*}}} .
$$

It is well known from [32] that the infimum in the formula above is attained at $\tilde{u}$, where

$$
\tilde{u}(x)=\frac{\kappa}{\left(\mu^{2}+\left|x-x_{0}\right|^{2}\right)^{\frac{N-2 s}{2}}}, \quad x \in \mathbb{R}^{N},
$$

with $\kappa \in \mathbb{R} \backslash\{0\}, \mu>0$ and $x_{0} \in \mathbb{R}^{N}$ fixed constants. We suppose $\kappa>0$ for our convenience. Equivalently, the function $\bar{u}$ defined as

$$
\bar{u}=\frac{\tilde{u}}{\|\tilde{u}\|_{L^{p}\left(\mathbb{R}^{N}\right)}}
$$

is such that

$$
S_{s}=\int_{\mathbb{R}^{2 N}}|\bar{u}(x)-\bar{u}(y)|^{2} K(x-y) d x d y .
$$

The function

$$
u^{*}(x)=\bar{u}\left(\frac{x}{S_{s}^{1 /(2 s)}}\right), \quad x \in \mathbb{R}^{N},
$$

is a solution of

$$
(-\Delta)^{s} u=|u|^{p-2} u \quad \text { in } \mathbb{R}^{N} .
$$

Now, we consider the family of functions $U_{\varepsilon}$ defined as

$$
U_{\varepsilon}(x)=\varepsilon^{-(N-2 s) / 2} u^{*}(x / \varepsilon), \quad x \in \mathbb{R}^{N},
$$

for any $\varepsilon>0$. The function $U_{\varepsilon}$ is a solution of problem (4.2) and satisfies

$$
\int_{\mathbb{R}^{2 N}}\left|U_{\varepsilon}(x)-U_{\varepsilon}(y)\right|^{2} K(x-y) d x d y=\int_{\mathbb{R}^{N}}\left|U_{\varepsilon}(x)\right|^{p} d x=S_{s}^{N /(2 s)} .
$$

Let us fix $\rho_{a}, \rho_{b}, \tilde{\rho}, \rho_{c}, \rho_{d}$ such that

$$
\rho_{1}<\rho_{a}<\rho_{b}<\tilde{\rho}<\rho_{c}<\rho_{d}<\rho_{2} .
$$

Let $\eta \in C_{0}^{\infty}\left(\mathbb{R}^{N}\right)$ be a radially symmetric function such that $0 \leq \eta \leq 1$ in $\mathbb{R}^{N}$ and

$$
\eta(x)= \begin{cases}0, & \text { if }|x| \leq \rho_{a} \\ 1, & \text { if } \rho_{b} \leq|x| \leq \rho_{c} \\ 0, & \text { if }|x| \geq \rho_{d}\end{cases}
$$


For every $\varepsilon \in(0,1)$ and $\mathbf{e} \in \mathbb{S}^{N-1}:=\left\{x \in \mathbb{R}^{N}:|x|=1\right\}$, we denote by $u_{\varepsilon, \mathbf{e}}$ the following function:

$$
u_{\varepsilon, \mathbf{e}}(x)=\eta(x) U_{\varepsilon}(x-\tilde{\rho} \mathbf{e}) .
$$

\section{Lemma 4.1 There hold}

(i) $\int_{\mathbb{R}^{N}}\left|u_{\varepsilon, \mathbf{e}}\right|^{2_{s}^{*}}=S_{s}^{\frac{N}{2 s}}+O\left(\varepsilon^{N}\right)$ uniformly in $\mathbf{e} \in \mathbb{S}^{N-1}$;

(ii) $\left\|u_{\varepsilon, \mathbf{e}}\right\|_{X_{0}}^{2}=S_{s}^{\frac{N}{2 s}}+O\left(\varepsilon^{N-2 s}\right)$ uniformly in $\mathbf{e} \in \mathbb{S}^{N-1}$.

Proof (i) By Proposition 22 in [32], we have

$$
\begin{aligned}
& \int_{\mathbb{R}^{N}}\left|u_{\varepsilon, \mathbf{e}}\right|^{2_{s}^{*}} d x \\
& \quad=\int_{\mathbb{R}^{N}} U_{\varepsilon}^{2_{s}^{*}}(x-\tilde{\rho} \mathbf{e}) d x+\int_{\mathbb{R}^{N}}\left(\eta^{2_{s}^{*}}(x)-1\right) U_{\varepsilon}^{2_{s}^{*}}(x-\tilde{\rho} \mathbf{e}) d x \\
& \quad=S_{s}^{\frac{N}{2 s}}+\int_{|x|<\rho_{b}}\left(\eta^{2_{s}^{*}}(x)-1\right) U_{\varepsilon}^{2_{s}^{*}}(x-\tilde{\rho} \mathbf{e}) d x+\int_{|x|>\rho_{c}}\left(\eta^{2_{s}^{*}}(x)-1\right) U_{\varepsilon}^{2_{s}^{*}}(x-\tilde{\rho} \mathbf{e}) d x .
\end{aligned}
$$

Direct computation yields that

$$
\begin{aligned}
& \left|\int_{|x|<\rho_{b}}\left(\eta^{2_{s}^{*}}(x)-1\right) U_{\varepsilon}^{2_{s}^{*}}(x-\tilde{\rho} \mathbf{e}) d x+\int_{|x|>\rho_{c}}\left(\eta^{2_{s}^{*}}(x)-1\right) U_{\varepsilon}^{2_{s}^{*}}(x-\tilde{\rho} \mathbf{e}) d x\right| \\
& \quad \leq C \varepsilon^{N}\left(\int_{|x|<\rho_{b}} \frac{d x}{|x-\tilde{\rho} \mathbf{e}|^{2 N}}+\int_{|x|>\rho_{c}} \frac{d x}{|x-\tilde{\rho} \mathbf{e}|^{2 N}}\right) \\
& \quad \leq C \varepsilon^{N}\left(\int_{|x|<\rho_{b}} \frac{d x}{\left(\tilde{\rho}-\rho_{b}\right)^{2 N}}+\int_{|x+\tilde{\rho} \mathbf{e}|>\rho_{c}} \frac{d x}{|x|^{2 N}}\right) \\
& \quad \leq C \varepsilon^{N}\left(\left(\tilde{\rho}-\rho_{b}\right)^{-2 N}\left|B_{\rho_{b}}(0)\right|+\int_{|x|>\rho_{c}-\tilde{\rho}} \frac{d x}{|x|^{2 N}}\right) \\
& \quad \leq C^{\prime} \varepsilon^{N} .
\end{aligned}
$$

Thus, by (4.8), we prove (i).

(ii) Set $\delta=\frac{1}{2} \min \left\{\tilde{\rho}-\rho_{b}, \rho_{c}-\tilde{\rho}\right\}$. Define

$$
\begin{aligned}
& \mathcal{D}_{1}=\left\{x \in \mathbb{R}^{N}: \rho_{b}<|x|<\rho_{c}\right\}, \\
& \mathcal{D}_{2}=\left\{x \in \mathbb{R}^{N}:|x| \leq \rho_{b} \text { or }|x| \geq \rho_{c}\right\}, \\
& \mathcal{D}_{3}=\left\{x \in \mathbb{R}^{N}:|x| \leq \rho_{a} \text { or }|x| \geq \rho_{d}\right\}, \\
& A_{1}=\left\{(x, y) \in \mathbb{R}^{N} \times \mathbb{R}^{N}: x \in \mathcal{D}_{1}, y \in \mathcal{D}_{1}\right\}, \\
& A_{2}=\left\{(x, y) \in \mathbb{R}^{N} \times \mathbb{R}^{N}: x \in \mathcal{D}_{1}, y \in \mathcal{D}_{2},|x-y|>\delta\right\}, \\
& A_{3}=\left\{(x, y) \in \mathbb{R}^{N} \times \mathbb{R}^{N}: x \in \mathcal{D}_{1}, y \in \mathcal{D}_{2},|x-y| \leq \delta\right\}, \\
& A_{4}=\left\{(x, y) \in \mathbb{R}^{N} \times \mathbb{R}^{N}: x \in \mathcal{D}_{2}, y \in \mathcal{D}_{2}\right\}, \\
& B_{1}=\left\{(x, y) \in \mathbb{R}^{N} \times \mathbb{R}^{N}:|x| \geq \rho_{c},|y| \geq \rho_{c},|t x+(1-t) y| \geq \rho_{c}, \forall t \in[0,1]\right\}, \\
& B_{2}=\left\{(x, y) \in \mathbb{R}^{N} \times \mathbb{R}^{N}:|x| \leq \rho_{b},|y| \leq \rho_{b},|t x+(1-t) y| \leq \rho_{b}, \forall t \in[0,1]\right\}, \\
& B_{3}=\left\{(x, y) \in \mathbb{R}^{N} \times \mathbb{R}^{N}: x \in \mathcal{D}_{3}, y \in \mathcal{D}_{3}\right\} .
\end{aligned}
$$


We have

$$
\begin{aligned}
\left\|u_{\varepsilon, \mathbf{e}}\right\|_{X_{0}}^{2} & =\int_{\mathbb{R}^{2 N}}\left|u_{\varepsilon, \mathbf{e}}(x)-u_{\varepsilon, \mathbf{e}}(y)\right|^{2} K(x-y) d x d y \\
& =\left(\int_{A_{1}}+2 \int_{A_{2}}+2 \int_{A_{3}}+\int_{A_{4}}\right)\left|u_{\varepsilon, \mathbf{e}}(x)-u_{\varepsilon, \mathbf{e}}(y)\right|^{2} K(x-y) d x d y .
\end{aligned}
$$

We consider the following four cases:

(i) Assume $(x, y) \in A_{4}$. Then $|x-\tilde{\rho} \mathbf{e}| \geq \tilde{\rho}-\rho_{b}$ or $|x-\tilde{\rho} \mathbf{e}| \geq \rho_{c}-\tilde{\rho}$. Thus, there exists constant $C>0$ such that

$$
\left|u_{\varepsilon, \mathbf{e}}(x)\right| \leq C \varepsilon^{-\frac{N-2 s}{2}}\left(\mu^{2}+\frac{|\xi-\tilde{\rho} \mathbf{e}|^{2}}{\left|\varepsilon S_{s}^{1 /(2 s)}\right|^{2}}\right)^{-\frac{N-2 s}{2}} \leq C \varepsilon^{\frac{N-2 s}{2}}
$$

Consequently,

$$
\left|u_{\varepsilon, \mathbf{e}}(x)-u_{\varepsilon, \mathbf{e}}(y)\right| \leq\left|u_{\varepsilon, \mathbf{e}}(x)\right|+\left|u_{\varepsilon, \mathbf{e}}(y)\right| \leq C \varepsilon^{\frac{N-2 s}{2}} .
$$

Moreover, if $(x, y) \in A_{4}$ and $|x-y| \leq \frac{1}{2}\left(\rho_{c}-\rho_{b}\right)$, then $(x, y) \in B_{1} \cup B_{2}$, and so $|\xi-\tilde{\rho} \mathbf{e}| \geq$ $\rho_{c}-\tilde{\rho}>0$ or $|\xi-\tilde{\rho} \mathbf{e}| \geq \tilde{\rho}-\rho_{b}>0$ for any $\xi$ on the segment joining $x$ and $y$. By the mean value theorem, there exists $\bar{\xi}$ on the segment joining $x$ and $y$ such that

$$
\begin{aligned}
\left|u_{\varepsilon, \mathbf{e}}(x)-u_{\varepsilon, \mathbf{e}}(y)\right| & =\left|\nabla u_{\varepsilon, \mathbf{e}}(\bar{\xi})\right| \cdot|x-y| \\
\leq & {\left[C \varepsilon^{-\frac{N-2 s}{2}}\left(\mu^{2}+\frac{|\bar{\xi}-\tilde{\rho} \mathbf{e}|^{2}}{\left|\varepsilon S_{s}^{1 /(2 s)}\right|^{2}}\right)^{-\frac{N-2 s}{2}}\right.} \\
& \left.+C \varepsilon^{-\frac{N-2 s}{2}}\left(\mu^{2}+\frac{|\bar{\xi}-\tilde{\rho} \mathbf{e}|^{2}}{\left|\varepsilon S_{s}^{1 /(2 s)}\right|^{2}}\right)^{-\frac{N-2 s}{2}-1} \frac{|\bar{\xi}-\tilde{\rho} \mathbf{e}|}{\left|\varepsilon S_{s}^{1 /(2 s)}\right|^{2}}\right]|x-y| \\
\leq & C \varepsilon^{\frac{N-2 s}{2}}|x-y| .
\end{aligned}
$$

Hence, by (4.11) and the inequality above, we get

$$
\left|u_{\varepsilon, \mathbf{e}}(x)-u_{\varepsilon, \mathbf{e}}(y)\right| \leq \begin{cases}C \varepsilon^{\frac{N-2 s}{2}}|x-y|, & \text { if }(x, y) \in A_{4} \text { and }|x-y| \leq \frac{1}{2}\left(\rho_{c}-\rho_{b}\right), \\ C \varepsilon^{\frac{N-2 s}{2}}, & \text { if }(x, y) \in A_{4} \text { and }|x-y|>\frac{1}{2}\left(\rho_{c}-\rho_{b}\right)\end{cases}
$$

or

$$
\left|u_{\varepsilon, \mathbf{e}}(x)-u_{\varepsilon, \mathbf{e}}(y)\right| \leq C \varepsilon^{\frac{N-2 s}{2}} \min \{1,|x-y|\} .
$$

Consequently, by the definition of $u_{\varepsilon, \mathbf{e}}$ and (4.13),

$$
\begin{aligned}
& \int_{A_{4}}\left|u_{\varepsilon, \mathbf{e}}(x)-u_{\varepsilon, \mathbf{e}}(y)\right|^{2} K(x-y) d x d y \\
& \quad=\int_{A_{4} \cap\left(\mathbb{R}^{2 N} \backslash B_{3}\right)}\left|u_{\varepsilon, \mathbf{e}}(x)-u_{\varepsilon, \mathbf{e}}(y)\right|^{2} K(x-y) d x d y
\end{aligned}
$$




$$
\begin{aligned}
& \leq 2 \int_{A_{4} \cap\left(\left\{(x, y):|x| \leq \rho_{d}, y \in \mathbb{R}^{N}\right\}\right)}\left|u_{\varepsilon, \mathbf{e}}(x)-u_{\varepsilon, \mathbf{e}}(y)\right|^{2} K(x-y) d x d y \\
& \leq C \varepsilon^{N-2 s} \int_{A_{4} \cap\left(\left\{(x, y):|x| \leq \rho_{d}, y \in \mathbb{R}^{N}\right\}\right)} \frac{\min \left\{1,|x-y|^{2}\right\}}{|x-y|^{N+2 s}} d x d y \\
& \leq C \varepsilon^{N-2 s} \int_{\left\{(x, y):|x| \leq \rho_{d}, y \in \mathbb{R}^{N}\right\}} \frac{\min \left\{1,|x-y|^{2}\right\}}{|x-y|^{N+2 s}} d x d y \\
& =C \varepsilon^{N-2 s} \int_{|x| \leq \rho_{d}} d x \int_{\mathbb{R}^{N}} \frac{\min \left\{1,|y|^{2}\right\}}{|y|^{N-2 s}} d y \\
& =C \varepsilon^{N-2 s} \int_{|x| \leq \rho_{d}} d x\left(\int_{|y| \leq 1} \frac{|y|^{2}}{|y|^{N+2 s}} d y+\int_{|y|>1} \frac{1}{|y|^{N+2 s}} d y\right) \\
& \leq C \varepsilon^{N-2 s} .
\end{aligned}
$$

(ii) Assume $(x, y) \in A_{3}$. Let $\xi=t x+(1-t) y=y+t(x-y)$ for any $t \in[0,1]$. If $|y| \geq \rho_{c}$, then

$$
|\xi|=|y+t(x-y)| \geq|y|-|x-y| \geq \rho_{c}-\frac{1}{2}\left(\rho_{c}-\tilde{\rho}\right)=\frac{1}{2}\left(\rho_{c}+\tilde{\rho}\right),
$$

and so

$$
|\xi-\tilde{\rho} \mathbf{e}| \geq \frac{1}{2}\left(\rho_{c}+\tilde{\rho}\right)-\tilde{\rho}=\frac{1}{2}\left(\rho_{c}-\tilde{\rho}\right)>0 .
$$

If $|y| \leq \rho_{b}$, then

$$
|\xi| \leq|y|+|x-y| \leq \rho_{b}+\frac{1}{2}\left(\tilde{\rho}-\rho_{b}\right)=\frac{1}{2}\left(\tilde{\rho}+\rho_{b}\right),
$$

and so

$$
|\xi-\tilde{\rho} \mathbf{e}| \geq \tilde{\rho}-|\xi| \geq \tilde{\rho}-\frac{1}{2}\left(\tilde{\rho}+\rho_{b}\right)=\frac{1}{2}\left(\tilde{\rho}-\rho_{b}\right)>0 .
$$

Thus, by the mean value theorem, there exists $\bar{\xi}$ on the segment joining $x$ and $y$ such that

$$
\left|u_{\varepsilon, \mathbf{e}}(x)-u_{\varepsilon, \mathbf{e}}(y)\right|=\left|\nabla u_{\varepsilon, \mathbf{e}}(\bar{\xi})\right| \cdot|x-y| \leq C \varepsilon^{\frac{N-2 s}{2}}|x-y| .
$$

\section{Consequently,}

$$
\begin{aligned}
& \int_{A_{3}}\left|u_{\varepsilon, \mathbf{e}}(x)-u_{\varepsilon, \mathbf{e}}(y)\right|^{2} K(x-y) d x d y \\
& \quad \leq C \varepsilon^{N-2 s} \int_{A_{3}} \frac{|x-y|^{2}}{|x-y|^{N+2 s}} d x d y \\
& \quad \leq C \varepsilon^{\frac{N-2 s}{2}} \int_{\tilde{A}_{3}} \frac{|x-y|^{2}}{|x-y|^{N+2 s}} d x d y \\
& \quad \leq C \varepsilon^{N-2 s} \int_{\mathcal{D}_{1}} d x \int_{\left\{y \in \mathbb{R}^{N}:|x-y| \leq \delta\right\}} \frac{1}{|x-y|^{N+2 s-2}} d y \\
& \quad=C \varepsilon^{N-2 s} \int_{\mathcal{D}_{1}} d x \int_{|y| \leq \delta} \frac{1}{|y|^{N+2 s-2}} d y \\
& \quad \leq C \varepsilon^{N-2 s}
\end{aligned}
$$

where $\tilde{A}_{3}=\left\{(x, y) \in \mathbb{R}^{N} \times \mathbb{R}^{N}: x \in \mathcal{D}_{1}, y \in \mathbb{R}^{N},|x-y| \leq \delta\right\}$. 
(iii) Assume $(x, y) \in A_{2}$. Since $x \in \mathcal{D}_{1}$, we have

$$
\begin{aligned}
& \int_{A_{2}}\left|u_{\varepsilon, \mathbf{e}}(x)-u_{\varepsilon, \mathbf{e}}(y)\right|^{2} K(x-y) d x d y \\
& =\int_{A_{2}}\left|U_{\varepsilon}(x-\tilde{\rho} \mathbf{e})-u_{\varepsilon, \mathbf{e}}(y)\right|^{2} K(x-y) d x d y \\
& =\int_{A_{2}}\left|U_{\varepsilon}(x-\tilde{\rho} \mathbf{e})-U_{\varepsilon}(y-\tilde{\rho} \mathbf{e})+U_{\varepsilon}(y-\tilde{\rho} \mathbf{e})-u_{\varepsilon, \mathbf{e}}(y)\right|^{2} K(x-y) d x d y \\
& \leq \int_{A_{2}}\left|U_{\varepsilon}(x-\tilde{\rho} \mathbf{e})-U_{\varepsilon}(y-\tilde{\rho} \mathbf{e})\right|^{2} K(x-y) d x d y \\
& \quad+\int_{A_{2}}\left|U_{\varepsilon}(y-\tilde{\rho} \mathbf{e})-u_{\varepsilon, \mathbf{e}}(y)\right|^{2} K(x-y) d x d y \\
& \quad+2 \int_{A_{2}}\left|U_{\varepsilon}(x-\tilde{\rho} \mathbf{e})-U_{\varepsilon}(y-\tilde{\rho} \mathbf{e})\right| \cdot\left|U_{\varepsilon}(y-\tilde{\rho} \mathbf{e})-u_{\varepsilon, \mathbf{e}}(y)\right| K(x-y) d x d y .
\end{aligned}
$$

Direct computation yields

$$
\begin{aligned}
& \int_{A_{2}}\left|U_{\varepsilon}(y-\tilde{\rho} \mathbf{e})-u_{\varepsilon, \mathbf{e}}(y)\right|^{2} K(x-y) d x d y \\
& \quad \leq \int_{A_{2}} \frac{\left(\left|U_{\varepsilon}(y-\tilde{\rho} \mathbf{e})\right|+\left|u_{\varepsilon, \mathbf{e}}(y)\right|\right)^{2}}{|x-y|^{N+2 s}} d x d y \\
& \quad \leq 4 \int_{A_{2}} \frac{\left|U_{\varepsilon}(y-\tilde{\rho} \mathbf{e})\right|^{2}}{|x-y|^{N+2 s}} d x d y \\
& \leq C \varepsilon^{N-2 s} \int_{A_{2}} \frac{1}{|x-y|^{N+2 s}} d x d y \\
& \quad=C \varepsilon^{N-2 s} \int_{\mathcal{D}_{1}} d x \int_{\left\{y \in \mathbb{R}^{N}:|x-y|>\delta\right\}} \frac{1}{|x-y|^{N+2 s}} d y \\
& \leq C \varepsilon^{N-2 s} \int_{\mathcal{D}_{1}} d x \int_{\left\{y \in \mathbb{R}^{N}:|y|>\delta\right\}} \frac{1}{|y|^{N+2 s}} d y \\
& \leq C \varepsilon^{N-2 s},
\end{aligned}
$$

where $\tilde{A}_{2}=\left\{(x, y) \in \mathbb{R}^{N} \times \mathbb{R}^{N}: x \in \mathcal{D}_{1}, y \in \mathbb{R}^{N},|x-y|>\delta\right\}$.

For any $(x, y) \in A_{2}$,

$$
\left|U_{\varepsilon}(x-\tilde{\rho} \mathbf{e}) U_{\varepsilon}(y-\tilde{\rho} \mathbf{e})\right| \leq C \varepsilon^{\frac{N-2 s}{2}}\left|U_{\varepsilon}(x-\tilde{\rho} \mathbf{e})\right| \leq C\left(\mu^{2}+\left|\frac{x-\tilde{\rho} \mathbf{e}}{\varepsilon S_{s}^{1 /(2 s)}}\right|^{2}\right)^{-\frac{N-2 s}{2}}
$$

Therefore, using the change of variable $\xi=x, \zeta=x-y$, we have that

$$
\begin{aligned}
& \int_{A_{2}}\left|U_{\varepsilon}(x-\tilde{\rho} \mathbf{e})\right| \cdot\left|U_{\varepsilon}(y-\tilde{\rho} \mathbf{e})-u_{\varepsilon, \mathbf{e}}(y)\right| K(x-y) d x d y \\
& \quad \leq 2 \int_{A_{2}}\left|U_{\varepsilon}(x-\tilde{\rho} \mathbf{e})\right| \cdot\left|U_{\varepsilon}(y-\tilde{\rho} \mathbf{e})\right| K(x-y) d x d y \\
& \quad \leq C \int_{A_{2}}\left(\mu^{2}+\left|\frac{x-\tilde{\rho} \mathbf{e}}{\varepsilon S_{s}^{1 /(2 s)}}\right|^{2}\right)^{-\frac{N-2 s}{2}}|x-y|^{-(N+2 s)} d x d y
\end{aligned}
$$




$$
\begin{aligned}
& =C \int_{\mathcal{D}_{1}}\left(\mu^{2}+\left|\frac{\xi-\tilde{\rho} \mathbf{e}}{\varepsilon S_{s}^{1 /(2 s)}}\right|^{2}\right)^{-\frac{N-2 s}{2}} d \xi \int_{\left\{\zeta \in \mathbb{R}^{N}:|\zeta|>\delta\right\}} \frac{1}{\zeta^{N+2 s}} d \zeta \\
& \leq C \int_{\left\{\xi \in \mathbb{R}^{N}:|\xi-\tilde{\rho} \mathbf{e}| \leq \rho_{c}+\tilde{\rho}\right\}}\left(\mu^{2}+\left|\frac{\xi-\tilde{\rho} \mathbf{e}}{\varepsilon S_{s}^{1 /(2 s)}}\right|^{2}\right)^{-\frac{N-2 s}{2}} d \xi \\
& \leq C \varepsilon^{N} \int_{\left\{\xi \in \mathbb{R}^{N}:|\xi| \leq S_{s}^{-1 /(2 s)}\left(\rho_{c}+\tilde{\rho}\right) \varepsilon^{-1}\right\}}\left(\mu^{2}+|\xi|^{2}\right)^{-\frac{N-2 s}{2}} d \xi \\
& \leq C \varepsilon^{N}\left(\int_{\left\{x \in \mathbb{R}^{N}:|\xi| \leq 1\right\}}+\int_{\left\{x \in \mathbb{R}^{N}: 1 \leq|\xi| \leq S_{s}^{-1 /(2 s)}\left(\rho_{c}+\tilde{\rho}\right) \varepsilon^{-1}\right\}}\right)\left(\mu^{2}+|\xi|^{2}\right)^{-\frac{N-2 s}{2}} d \xi \\
& \leq C \varepsilon^{N-2 s .}
\end{aligned}
$$

Similar to (4.17), we have

$$
\begin{aligned}
& \int_{A_{2}}\left|U_{\varepsilon}(y-\tilde{\rho} \mathbf{e})\right| \cdot\left|U_{\varepsilon}(y-\tilde{\rho} \mathbf{e})-u_{\varepsilon, \mathbf{e}}(y)\right| K(x-y) d x d y \\
& \quad \leq 2 \int_{A_{2}} \frac{\left|U_{\varepsilon}(y-\tilde{\rho} \mathbf{e})\right|^{2}}{|x-y|^{N+2 s}} d x d y \\
& \quad \leq C \varepsilon^{N-2 s} .
\end{aligned}
$$

By (4.16)-(4.19), we get

$$
\begin{aligned}
& \int_{A_{2}}\left|u_{\varepsilon, \mathbf{e}}(x)-u_{\varepsilon, \mathbf{e}}(y)\right|^{2} K(x-y) d x d y \\
& \quad \leq \int_{A_{2}}\left|U_{\varepsilon}(x-\tilde{\rho} \mathbf{e})-U_{\varepsilon}(y-\tilde{\rho} \mathbf{e})\right|^{2} K(x-y) d x d y+C \varepsilon^{N-2 s} .
\end{aligned}
$$

By (4.10), (4.14), (4.15), and (4.20), we have

$$
\begin{aligned}
& \int_{\mathbb{R}^{2 N}}\left|u_{\varepsilon, \mathbf{e}}(x)-u_{\varepsilon, \mathbf{e}}(y)\right|^{2} K(x-y) d x d y \\
& \leq \int_{A_{1}}\left|u_{\varepsilon, \mathbf{e}}(x)-u_{\varepsilon, \mathbf{e}}(y)\right|^{2} K(x-y) d x d y \\
& \quad+2 \int_{A_{2}}\left|U_{\varepsilon}(x-\tilde{\rho} \mathbf{e})-U_{\varepsilon}(y-\tilde{\rho} \mathbf{e})\right|^{2} K(x-y) d x d y+C \varepsilon^{N-2 s} \\
& \leq \int_{\mathbb{R}^{2 N}}\left|U_{\varepsilon}(x-\tilde{\rho} \mathbf{e})-U_{\varepsilon}(y-\tilde{\rho} \mathbf{e})\right|^{2} K(x-y) d x d y+C \varepsilon^{N-2 s} .
\end{aligned}
$$

Using the change of variable and (4.13) in [32], we have

$$
\begin{aligned}
\int_{\mathbb{R}^{2 N}}\left|U_{\varepsilon}(x-\tilde{\rho} \mathbf{e})-U_{\varepsilon}(y-\tilde{\rho} \mathbf{e})\right|^{2} K(x-y) d x d y & =\int_{\mathbb{R}^{2 N}}\left|U_{\varepsilon}(x)-U_{\varepsilon}(y)\right|^{2} K(x-y) d x d y \\
& =\int_{\mathbb{R}^{N}}\left|U_{\varepsilon}(x)\right|^{2_{s}^{*}} d x=S_{s}^{\frac{N}{2 s}}
\end{aligned}
$$

By (4.21) and (4.22), we have

$$
\left\|u_{\varepsilon, \mathbf{e}}\right\|_{X_{0}}^{2}=\int_{\mathbb{R}^{2 N}}\left|u_{\varepsilon, \mathbf{e}}(x)-u_{\varepsilon, \mathbf{e}}(y)\right|^{2} K(x-y) d x d y \leq S_{s}^{\frac{N}{2 s}}+C \varepsilon^{N-2 s} .
$$


On the other hand, by the definition of $S_{s}$ and (i), we have

$$
\left\|u_{\varepsilon, \mathbf{e}}\right\|_{X_{0}}^{2} \geq S_{s}\left(\int_{\mathbb{R}^{N}}\left|u_{\varepsilon, \mathbf{e}}\right|^{2_{s}^{*}} d x\right)^{2 / 2_{s}^{*}}=S_{s}^{\frac{N}{2 s}}+o(1) .
$$

Combining (4.23) and (4.24), we prove (ii).

Lemma 4.2 Assume that $a \in C(\bar{\Omega})$ with $\left|a^{+}\right|_{q} \in\left(0, \sigma_{2}\right)$. There exists $\varepsilon_{0}>0$ such that, for $\varepsilon<\varepsilon_{0}$,

$$
\sup _{t \geq 0} I\left(\tilde{u}_{1}+t u_{\varepsilon, \mathbf{e}}\right)<m^{+}+\frac{s}{N} S_{s}^{\frac{N}{2 s}}
$$

uniformly in $\mathbf{e} \in \mathbb{S}^{N-1}$, where $\tilde{u}_{1}$ is a minimizer of I in Theorem 3.3.

Proof Since $I$ is continuous in $X_{0}$ and $u_{\varepsilon, \mathbf{e}}$ is uniformly bounded in $X_{0}$ for $\varepsilon$ small enough, there exists $t_{1}>0$ such that, for $t \in\left[0, t_{1}\right]$,

$$
I\left(\tilde{u}_{1}+t u_{\varepsilon, \mathbf{e}}\right)<I\left(\tilde{u}_{1}\right)+\frac{s}{N} S_{S}^{\frac{N}{2 s}} .
$$

Since $u_{\varepsilon, \mathbf{e}}(x)=0$ for any $x \in\{x \in \Omega: a(x)<0\}$, we have

$$
\begin{aligned}
I\left(\tilde{u}_{1}+t u_{\varepsilon, \mathbf{e}}\right)= & \frac{1}{2}\left\|\tilde{u}_{1}+t u_{\varepsilon, \mathbf{e}}\right\|_{X_{0}}^{2}-\frac{1}{p} \int_{\Omega} a(x)\left(\tilde{u}_{1}+t u_{\varepsilon, \mathbf{e}}\right)^{p} d x-\frac{1}{2_{s}^{*}} \int_{\Omega}\left(\tilde{u}_{1}+t u_{\varepsilon, \mathbf{e}}\right)^{2_{s}^{*}} d x \\
= & \frac{1}{2}\left\|\tilde{u}_{1}\right\|_{X_{0}}^{2}+t\left(\tilde{u}_{1}, u_{\varepsilon, \mathbf{e}}\right)_{X_{0}}+\frac{t^{2}}{2}\left\|u_{\varepsilon, \mathbf{e}}\right\|_{X_{0}}^{2}-\frac{1}{p} \int_{\Omega} a^{+}(x)\left(\tilde{u}_{1}+t u_{\varepsilon, \mathbf{e}}\right)^{p} d x \\
& +\frac{1}{p} \int_{\Omega} a^{-}(x) \tilde{u}_{1}^{p} d x-\frac{1}{2_{s}^{*}} \int_{\Omega}\left(\tilde{u}_{1}+t u_{\varepsilon, \mathbf{e}}\right)^{2_{s}^{*}} d x .
\end{aligned}
$$

It is easy to get from Lemma 4.1 that

$$
\int_{\Omega} u_{\varepsilon, \mathbf{e}}^{2_{s}^{*}} d x \geq \frac{1}{2} S_{s}^{\frac{N}{2 s}}
$$

for $\varepsilon$ small enough. Note that the last term in (4.25) satisfies

$$
\frac{1}{2_{s}^{*}} \int_{\Omega}\left(\tilde{u}_{1}+t u_{\varepsilon, \mathbf{e}}\right)^{2_{s}^{*}} d x \geq \frac{t^{2_{s}^{*}}}{2_{s}^{*}} \int_{\Omega} u_{\varepsilon, \mathbf{e}}^{2_{s}^{*}} d x \geq \frac{S_{s}^{\frac{N}{2 s}}}{22_{s}^{*}} t^{2_{s}^{*}}
$$

Thus, $I\left(\tilde{u}_{1}+t u_{\varepsilon, \mathbf{e}}\right) \rightarrow-\infty$ as $t \rightarrow+\infty$ uniformly in $\varepsilon$ and e. Consequently, there exists $t_{2}>t_{1}$ such that $I\left(\tilde{u}_{1}+t u_{\varepsilon, \mathbf{e}}\right)<m^{+}+\frac{s}{N} S_{s}^{\frac{N}{2 s}}$ for $t \geq t_{2}$. Then, we only need to verify the inequality

$$
\sup _{t_{1} \leq t \leq t_{2}} I\left(\tilde{u}_{1}+t u_{\varepsilon, \mathbf{e}}\right)<m^{+}+\frac{s}{N} S_{s}^{\frac{N}{2 s}}
$$

for $\varepsilon$ small enough.

From now on, we assume that $t \in\left[t_{1}, t_{2}\right]$. 
There exists a constant $C>0$ such that

$$
\begin{aligned}
\int_{\Omega}\left(\tilde{u}_{1}+t u_{\varepsilon, \mathbf{e}}\right)^{2_{s}^{*}} d x \geq & \int_{\Omega} \tilde{u}_{1}^{2_{s}^{*}} d x+t^{2_{s}^{*}} \int_{\Omega} u_{\varepsilon, \mathbf{e}}^{2_{s}^{*}} d x+2_{s}^{*} t \int_{\Omega} \tilde{u}_{1}^{2_{s}^{*}-1} u_{\varepsilon, \mathbf{e}} d x \\
& +2_{s}^{*} t^{2_{s}^{*}-1} \int_{\Omega} u_{\varepsilon, \mathbf{e}}^{2_{s}^{*}-1} \tilde{u}_{1} d x-C t^{2_{s}^{*} / 2} \int_{\Omega} \tilde{u}_{1}^{2_{s}^{*} / 2} u_{\varepsilon, \mathbf{e}}^{2_{s}^{*} / 2} d x .
\end{aligned}
$$

We have used the following inequality (see $[5,40]$ for example): for $r>2$, there exists a constant $C_{r}$ (depending on $r$ ) such that

$$
(\alpha+\beta)^{r} \geq \alpha^{r}+\beta^{r}+r\left(\alpha^{r-1} \beta+\alpha \beta^{r-1}\right)-C_{r} \alpha^{r / 2} \beta^{r / 2} \quad \forall \alpha, \beta>0 .
$$

Combining (4.25) and (4.26), and using the fact that $\tilde{u}_{1}$ is a positive solution of (1.1), we have

$$
\begin{aligned}
& I\left(\tilde{u}_{1}+t u_{\varepsilon, \mathbf{e}}\right) \\
& \leq \frac{1}{2}\left\|\tilde{u}_{1}\right\|_{X_{0}}^{2}+t\left(\tilde{u}_{1}, u_{\varepsilon, \mathbf{e}}\right)_{X_{0}}+\frac{t^{2}}{2}\left\|u_{\varepsilon, \mathbf{e}}\right\|_{X_{0}}^{2}-\frac{1}{p} \int_{\Omega} a^{+}(x)\left(\tilde{u}_{1}+t u_{\varepsilon, \mathbf{e}}\right)^{p} d x \\
& +\frac{1}{p} \int_{\Omega} a^{-}(x) \tilde{u}_{1}^{p} d x-\frac{1}{2_{s}^{*}} \int_{\Omega} \tilde{u}_{1}^{2_{s}^{*}} d x-\frac{1}{2_{s}^{*}} t^{2_{s}^{*}} \int_{\Omega} u_{\varepsilon, \mathrm{e}}^{2_{s}^{*}} d x \\
& -t \int_{\Omega} \tilde{u}_{1}^{2_{s}^{*}-1} u_{\varepsilon, \mathbf{e}} d x-t^{2_{s}^{*}-1} \int_{\Omega} u_{\varepsilon, \mathbf{e}}^{2_{s}^{*}-1} \tilde{u}_{1} d x+C t^{2_{s}^{*} / 2} \int_{\Omega} \tilde{u}_{1}^{2_{s}^{*} / 2} u_{\varepsilon, \mathbf{e}}^{2_{s}^{*} / 2} d x \\
& =\frac{1}{2}\left\|\tilde{u}_{1}\right\|_{X_{0}}^{2}+t \int_{\Omega} a(x)^{+} \tilde{u}_{1}^{p-1} u_{\varepsilon, \mathbf{e}} d x+\frac{t^{2}}{2}\left\|u_{\varepsilon, \mathbf{e}}\right\|_{X_{0}}^{2}-\frac{1}{p} \int_{\Omega} a^{+}(x)\left(\tilde{u}_{1}+t u_{\varepsilon, \mathbf{e}}\right)^{p} d x \\
& +\frac{1}{p} \int_{\Omega} a^{-}(x) \tilde{u}_{1}^{p} d x-\frac{1}{2_{s}^{*}} \int_{\Omega} \tilde{u}_{1}^{2_{s}^{*}} d x-\frac{1}{2_{s}^{*}} t^{2_{s}^{*}} \int_{\Omega} u_{\varepsilon, \mathbf{e}}^{2_{s}^{*}} d x-t^{2_{s}^{*}-1} \int_{\Omega} u_{\varepsilon, \mathbf{e}}^{2_{s}^{*}-1} \tilde{u}_{1} d x \\
& +C t^{2_{s}^{*} / 2} \int_{\Omega} \tilde{u}_{1}^{2_{s}^{*} / 2} u_{\varepsilon, \mathbf{e}}^{2_{s}^{*} / 2} d x \\
& =I\left(\tilde{u}_{1}\right)+t \int_{\Omega} a^{+}(x) \tilde{u}_{1}^{p-1} u_{\varepsilon, \mathbf{e}} d x+\frac{t^{2}}{2}\left\|u_{\varepsilon, \mathbf{e}}\right\|_{X_{0}}^{2}-\frac{1}{p} \int_{\Omega} a^{+}(x)\left(\tilde{u}_{1}+t u_{\varepsilon, \mathbf{e}}\right)^{p} d x \\
& +\frac{1}{p} \int_{\Omega} a^{+}(x) \tilde{u}_{1}^{p} d x-\frac{1}{2_{s}^{*}} t^{2_{s}^{*}} \int_{\Omega} u_{\varepsilon, \mathbf{e}}^{2_{s}^{*}} d x-t^{2_{s}^{*}-1} \int_{\Omega} u_{\varepsilon, \mathbf{e}}^{2_{s}^{*}-1} \tilde{u}_{1} d x \\
& +C t^{2_{s}^{*} / 2} \int_{\Omega} \tilde{u}_{1}^{2_{s}^{*} / 2} u_{\varepsilon, \mathbf{e}}^{2_{s}^{*} / 2} d x \\
& =I\left(\tilde{u}_{1}\right)+\frac{t^{2}}{2}\left\|u_{\varepsilon, \mathbf{e}}\right\|_{X_{0}}^{2}-\frac{1}{2_{s}^{*}} t^{2_{s}^{*}} \int_{\Omega} u_{\varepsilon, \mathbf{e}}^{2_{s}^{*}} d x \\
& -\frac{1}{p} \int_{\Omega} a^{+}(x)\left[\left(\tilde{u}_{1}+t u_{\varepsilon, \mathbf{e}}\right)^{p} d x-\tilde{u}_{1}^{p}-p \tilde{u}_{1}^{p-1} t u_{\varepsilon, \mathbf{e}}\right] d x \\
& -t^{2_{s}^{*}-1} \int_{\Omega} u_{\varepsilon, \mathbf{e}}^{2_{s}^{*}-1} \tilde{u}_{1} d x+C t^{2_{s}^{*} / 2} \int_{\Omega} \tilde{u}_{1}^{2_{s}^{*} / 2} u_{\varepsilon, \mathbf{e}}^{2_{s}^{*} / 2} d x \\
& \leq I\left(\tilde{u}_{1}\right)+\frac{t^{2}}{2}\left\|u_{\varepsilon, \mathbf{e}}\right\|_{X_{0}}^{2}-\frac{t^{2_{s}^{*}}}{2_{s}^{*}} \int_{\Omega} u_{\varepsilon, \mathbf{e}}^{2_{s}^{*}} d x \\
& -t^{2_{s}^{*} / 2}\left(t^{\left(2_{s}^{*}-2\right) / 2} \int_{\Omega} u_{\varepsilon, \mathbf{e}}^{2_{s}^{*}-1} \tilde{u}_{1} d x-C \int_{\Omega} \tilde{u}_{1}^{2_{s}^{*} / 2} u_{\varepsilon, \mathbf{e}}^{2_{s}^{*} / 2} d x\right) \\
& \leq I\left(\tilde{u}_{1}\right)+S_{s}^{\frac{N}{2 s}}\left(\frac{t^{2}}{2}-\frac{t^{2 *}}{2_{s}^{*}}\right)-t^{\frac{2_{s}^{*}}{2}}\left(t^{2_{s}^{*}-2} \int_{\Omega} u_{\varepsilon, \mathbf{e}}^{2_{s}^{*}-1} \tilde{u}_{1} d x-C \int_{\Omega} \tilde{u}_{1}^{2_{s}^{*} / 2} u_{\varepsilon, \mathbf{e}}^{2_{s}^{*} / 2} d x\right)
\end{aligned}
$$




$$
\begin{aligned}
& +O\left(\varepsilon^{N-2 s}\right) \\
\leq & I\left(\tilde{u}_{1}\right)+\frac{s}{N} S_{s}^{\frac{N}{2 s}}-t^{2_{s}^{*} / 2}\left(t^{\frac{2 s}{2}-2} \int_{\Omega} u_{\varepsilon, \mathbf{e}}^{2_{s}^{*}-1} \tilde{u}_{1} d x-C \int_{\Omega} \tilde{u}_{1}^{2_{s}^{*} / 2} u_{\varepsilon, \mathbf{e}}^{2_{s}^{*} / 2} d x\right) \\
& +O\left(\varepsilon^{N-2 s}\right) .
\end{aligned}
$$

Here we have used the elementary inequality: $(\alpha+\beta)^{p} \geq \alpha^{p}+p \alpha^{p-1} \beta, \forall \alpha, \beta>0$.

Now, we estimate the last but one term in (4.27). By Theorem 3.3, there exists a constant $C_{1}>0$ such that $\tilde{u}_{1}(x) \geq C_{1}$ for $x \in E:=\left\{x \in \mathbb{R}^{N}: \rho_{b} \leq|x| \leq \rho_{c}\right\}$. Thus,

$$
\begin{aligned}
\int_{\Omega} u_{\varepsilon, \mathbf{e}}^{2_{s}^{*}-1} \tilde{u}_{1} d x & \geq C_{1} \int_{E} u_{\varepsilon, \mathbf{e}}^{2_{s}^{*}-1} d x \\
& \geq C_{1} \int_{E} U_{\varepsilon}^{2_{s}^{*}-1}(x-\tilde{\rho} \mathbf{e}) d x \\
& \geq C_{1} \int_{E_{1}} U_{\varepsilon}^{2_{s}^{*}-1}(x) d x \\
& \geq C_{1} \varepsilon^{\frac{N-2 s}{2}} \int_{E_{2}} \frac{d x}{\left(\mu^{2}+|x|^{2}\right)^{\frac{N+2 s}{2}}} \\
& \geq C_{2} \varepsilon^{\frac{N-2 s}{2}}
\end{aligned}
$$

for $\varepsilon$ small enough, where

$$
\begin{aligned}
& E_{1}:=\left\{x \in \mathbb{R}^{N}:|x| \leq \min \left\{\tilde{\rho}-\rho_{b}, \rho_{c}-\tilde{\rho}\right\}\right\}, \\
& E_{2}:=\left\{x \in \mathbb{R}^{N}:|x| \leq \min \left\{\tilde{\rho}-\rho_{b}, \rho_{c}-\tilde{\rho}\right\} / \varepsilon\right\} .
\end{aligned}
$$

Direct computation yields that

$$
\begin{aligned}
\int_{\Omega} \tilde{u}_{1}^{2_{s}^{*} / 2} u_{\varepsilon, \mathbf{e}}^{2_{s}^{*} / 2} d x & \leq C_{3} \int_{\Omega} u_{\varepsilon, \mathbf{e}}^{2_{s}^{*} / 2} d x \\
& \leq C_{3} \int_{D_{1}} U_{\varepsilon}^{2_{s}^{*} / 2}(x-\tilde{\rho} \mathbf{e}) d x \\
& \leq C_{3} \int_{D_{2}} U_{\varepsilon}^{2_{s}^{*} / 2}(x) d x \\
& \leq C_{4} \varepsilon^{\frac{N}{2}} \int_{D_{3}} \frac{1}{\left(\mu^{2}+|x|^{2}\right)^{N / 2}} d x \\
& \leq C_{5} \varepsilon^{\frac{N}{2}}|\ln \varepsilon|
\end{aligned}
$$

where

$$
\begin{aligned}
& D_{1}:=\left\{x \in \mathbb{R}^{N}: \rho_{a} \leq|x| \leq \rho_{d}\right\}, \\
& D_{2}:=\left\{x \in \mathbb{R}^{N}:|x| \leq \rho_{d}+\tilde{\rho}\right\}, \\
& D_{3}:=\left\{x \in \mathbb{R}^{N}:|x| \leq\left(\rho_{d}+\tilde{\rho}\right) / \varepsilon\right\} .
\end{aligned}
$$


Hence, by (4.28) and (4.29), we have

$$
\begin{aligned}
& t^{\left(2_{s}^{*}-2\right) / 2} \int_{\Omega} u_{\varepsilon, \mathbf{e}}^{2_{s}^{*}-1} \tilde{u}_{1} d x-C \int_{\Omega} \tilde{u}_{1}^{2_{s}^{*} / 2} u_{\varepsilon, \mathbf{e}}^{2_{s}^{*} / 2} d x \\
& \geq t_{1}^{\frac{2_{s}^{*}-2}{2}} \int_{\Omega} u_{\varepsilon, \mathbf{e}}^{2_{s}^{*}-1} \tilde{u}_{1} d x-C \int_{\Omega} \tilde{u}_{1}^{2_{s}^{*} / 2} u_{\varepsilon, \mathbf{e}}^{2_{s}^{*} / 2} d x \\
& \geq t_{1}^{\frac{2_{s}^{*}-2}{2}} C_{2} \varepsilon^{\frac{N-2 s}{2}}-C_{5} \varepsilon^{\frac{N}{2}}|\ln \varepsilon|
\end{aligned}
$$

for $\varepsilon>0$ small enough. Consequently, by (4.27), we have

$$
\sup _{t_{1} \leq t \leq t_{2}} I\left(\tilde{u}_{1}+t u_{\varepsilon, \mathbf{e}}\right)<I\left(\tilde{u}_{1}\right)+\frac{s}{N} S_{s}^{\frac{N}{2 s}}
$$

for $\varepsilon>0$ small enough.

Let

$$
\begin{aligned}
& \mathcal{A}_{1}:=\left\{u \in X_{0}^{+}: \frac{1}{\|u\|_{X_{0}}} t^{-}\left(\frac{u}{\|u\|_{X_{0}}}\right)>1\right\}, \\
& \mathcal{A}_{2}:=\left\{u \in X_{0}^{+}: \frac{1}{\|u\|_{X_{0}}} t^{-}\left(\frac{u}{\|u\|_{X_{0}}}\right)<1\right\} .
\end{aligned}
$$

Lemma 4.3 Assume that $a \in C(\bar{\Omega})$ with $\left|a^{+}\right|_{q} \in\left(0, \sigma_{2}\right)$. We have

(i) $X_{0}^{+}=\mathcal{A}_{1} \cup \mathcal{A}_{2} \cup \mathcal{N}^{-}$;

(ii) $\mathcal{N}^{+} \subset \mathcal{A}_{1}$;

(iii) for each $\varepsilon<\varepsilon_{0}\left(\varepsilon_{0}\right.$ is defined in Lemma 4.2), there exists $t_{0}>1$ such that $\tilde{u}_{1}+t_{0} u_{\varepsilon, \mathbf{e}} \in \mathcal{A}_{2}$ for all $\mathbf{e} \in \mathbb{S}^{N-1}$

(iv) for each $\varepsilon<\varepsilon_{0}$, there exists $s_{0} \in(0,1)$ such that $\tilde{u}_{1}+s_{0} t_{0} u_{\varepsilon, \mathbf{e}} \in \mathcal{N}^{-}$for all $\mathbf{e} \in \mathbb{S}^{N-1}$;

(v) $m^{-}<m^{+}+\frac{s}{N} S_{s}^{\frac{N}{2 s}}$.

Proof (i) By Lemma 2.4(iv) we prove (i).

(ii) For any $u \in \mathcal{N}^{+}$, by (2.6), we get that $\int_{\Omega} a(x)\left(u^{+}\right)^{p} d x>0$. Let $v=\frac{u}{\|u\|_{X_{0}}}$. By Lemma 2.4, there exists $t^{-}(v)>0$ such that $t^{-}(v) v \in \mathcal{N}^{-}$, that is,

$$
\frac{1}{\|u\|_{X_{0}}} t^{-}\left(\frac{u}{\|u\|_{X_{0}}}\right) u \in \mathcal{N}^{-} .
$$

Hence,

$$
t^{-}(u)=\frac{1}{\|u\|_{X_{0}}} t^{-}\left(\frac{u}{\|u\|_{X_{0}}}\right) .
$$

By Lemma 2.4, we have

$$
1=t^{+}(u)<t_{\max }(u)<t^{-}(u) .
$$

Thus, we get $\mathcal{N}^{+} \subset \mathcal{A}_{1}$. 
(iii) We claim that there exists $C>0$ such that $\sup _{t \geq 0} t^{-}\left(\frac{\tilde{u}_{1}+t u_{\varepsilon, \mathbf{e}}}{\left\|\tilde{u}_{1}+t u_{\varepsilon, \mathbf{e}}\right\| X_{0}}\right)<C$. Assume by contradiction that there exists a sequence $\left\{t_{n}\right\}$ such that $t_{n} \rightarrow+\infty$ and $t^{-}\left(v_{n}\right) \rightarrow+\infty$ as $n \rightarrow \infty$, where $v_{n}:=\frac{\tilde{u}_{1}+t_{n} u_{\varepsilon, \mathbf{e}}}{\left\|\tilde{u}_{1}+t_{n} u_{\varepsilon, \mathbf{e}}\right\|_{X_{0}}}$. Since $t^{-}\left(v_{n}\right) v_{n} \in \mathcal{N}^{-}$, by Lebesgue's dominated convergence theorem, we have

$$
\int_{\Omega}\left(v_{n}^{+}\right)^{2_{s}^{*}} d x=\frac{1}{\left\|t_{n}^{-1} \tilde{u}_{1}+u_{\varepsilon, \mathbf{e}}\right\|_{X_{0}}^{2_{s}^{*}}} \int_{\Omega}\left(t_{n}^{-1} \tilde{u}_{1}+u_{\varepsilon, \mathbf{e}}\right)^{2_{s}^{*}} d x \rightarrow \frac{\int_{\Omega} u_{\varepsilon, \mathbf{e}}^{2_{s}^{*}} d x}{\left\|u_{\varepsilon, \mathbf{e}}\right\|_{X_{0}}^{2^{*}}}
$$

as $n \rightarrow \infty$. Thus,

$$
I\left(t^{-}\left(v_{n}\right) v_{n}\right)=\frac{1}{2}\left(t^{-}\left(v_{n}\right)\right)^{2}-\frac{\left(t^{-}\left(v_{n}\right)\right)^{p}}{p} \int_{\Omega} a(x)\left(v_{n}^{+}\right)^{p} d x-\frac{\left(t^{-}\left(v_{n}\right)\right)^{2_{s}^{*}}}{2_{s}^{*}} \int_{\Omega}\left(v_{n}^{+}\right)^{2_{s}^{*}} d x \rightarrow-\infty
$$

as $n \rightarrow \infty$, which is impossible since $I$ is bounded from below on $\mathcal{N}$ by Lemma 2.1. Set

$$
t_{0}=\frac{\left\|\tilde{u}_{1}\right\|_{X_{0}}+\left(\left\|\tilde{u}_{1}\right\|_{X_{0}}^{2}+\left|C^{2}-\left\|\tilde{u}_{1}\right\|_{X_{0}}^{2}\right|\right)^{1 / 2}}{\left\|u_{\varepsilon, \mathbf{e}}\right\|_{X_{0}}}+1
$$

Then

$$
\begin{aligned}
\left\|\tilde{u}_{1}+t_{0} u_{\varepsilon, \mathbf{e}}\right\|_{X_{0}}^{2} & =\left\|\tilde{u}_{1}\right\|_{X_{0}}^{2}+t_{0}^{2}\left\|u_{\varepsilon, \mathbf{e}}\right\|_{X_{0}}^{2}+2 t_{0}\left(\tilde{u}_{1}, u_{\varepsilon, \mathbf{e}}\right)_{X_{0}} \\
& >\left\|\tilde{u}_{1}\right\|_{X_{0}}^{2}+\left|C^{2}-\left\|\tilde{u}_{1}\right\|_{X_{0}}^{2}\right| \\
& \geq C^{2}>\left[t^{-}\left(\frac{\tilde{u}_{1}+t u_{\varepsilon, \mathbf{e}}}{\left\|\tilde{u}_{1}+t u_{\varepsilon, \mathbf{e}}\right\|_{X_{0}}}\right)\right]^{2} .
\end{aligned}
$$

Hence, we get $\tilde{u}_{1}+t_{0} u_{\varepsilon, \mathbf{e}} \in \mathcal{A}_{2}$.

(iv) Define $\gamma:[0,1] \rightarrow \mathbb{R}$ as

$$
\gamma(s):=\frac{1}{\left\|\tilde{u}_{1}+s t_{0} u_{\varepsilon, \mathbf{e}}\right\|_{X_{0}}} t^{-}\left(\frac{\tilde{u}_{1}+s t_{0} u_{\varepsilon, \mathbf{e}}}{\left\|\tilde{u}_{1}+s t_{0} u_{\varepsilon, \mathbf{e}}\right\|_{X_{0}}}\right) \quad \text { for all } s \in[0,1] .
$$

By Lemma 2.4(iii), $\gamma(s)$ is a continuous function of $s$. Since $\gamma(0)>1$ and $\gamma(1)<1$ there exists $s_{0} \in(0,1)$ such that $\gamma\left(s_{0}\right)=1$, that is, $\tilde{u}_{1}+s_{0} t_{0} u_{\varepsilon, \mathbf{e}} \in \mathcal{N}^{-}$.

(v) By Lemma 4.2 and (iv), we have $m^{-}<m^{+}+\frac{s}{N} S_{s}^{\frac{N}{2 s}}$.

Consider the following critical problem:

$$
\begin{cases}(-\Delta)^{s} u=|u|^{2_{s}^{*}-2} u & \text { in } \Omega, \\ u=0 & \text { in } \mathbb{R}^{N} \backslash \Omega .\end{cases}
$$

We define the energy functional $J: X_{0} \rightarrow \mathbb{R}$ associated with the critical problem (4.31) as

$$
J(u)=\frac{1}{2}\|u\|_{X_{0}}^{2}-\frac{1}{2_{s}^{*}} \int_{\Omega}|u|^{2_{s}^{*}} d x .
$$

Set

$$
\mathcal{M}(\Omega)=\left\{u \in X_{0} \backslash\{0\}:\left\langle J^{\prime}(u), u\right\rangle=0\right\}
$$


and

$$
\gamma(\Omega)=\inf _{u \in \mathcal{M}(\Omega)} J(u)
$$

Similarly, we define $J_{\infty}: \dot{H}^{s}\left(\mathbb{R}^{N}\right) \rightarrow \mathbb{R}$ as

$$
J_{\infty}(u)=\frac{1}{2} \int_{\mathbb{R}^{2 N}}(u(x)-u(y))^{2} K(x-y) d x d y-\frac{1}{2_{s}^{*}} \int_{\mathbb{R}^{N}}|u|^{2_{s}^{*}} d x,
$$

where $\dot{H}^{s}\left(\mathbb{R}^{N}\right)$ denotes the space of functions $u \in L^{p}\left(\mathbb{R}^{N}\right)$ such that $\int_{\mathbb{R}^{2 N}}(u(x)-u(y))^{2} K(x-$ y) $d x d y<\infty$. Set

$$
\mathcal{M}\left(\mathbb{R}^{N}\right)=\left\{u \in \dot{H}^{s}\left(\mathbb{R}^{N}\right):\left\langle J_{\infty}(u), u\right\rangle=0\right\}
$$

and

$$
\gamma\left(\mathbb{R}^{N}\right)=\inf _{u \in \mathcal{M}\left(\mathbb{R}^{N}\right)} J_{\infty}(u)
$$

It is easy to see that $\gamma\left(\mathbb{R}^{N}\right)=\frac{s}{N} S_{s}^{\frac{N}{2 s}}$.

Lemma 4.4 $\gamma(\Omega)=\gamma\left(\mathbb{R}^{N}\right)$ and $\gamma(\Omega)$ is never achieved except when $\Omega=\mathbb{R}^{N}$.

The proof of Lemma 4.4 can be found in [19], and we give a proof for the reader's' convenience although these results are known.

Proof Since $\mathcal{M}(\Omega) \subset \mathcal{M}\left(\mathbb{R}^{N}\right)$, we have $\gamma\left(\mathbb{R}^{N}\right) \leq \gamma(\Omega)$. Conversely, let $\left\{u_{n}\right\} \subset \dot{H}^{s}\left(\mathbb{R}^{N}\right)$ be a minimizing sequence for $\gamma\left(\mathbb{R}^{N}\right)$. By density of $C_{0}^{\infty}\left(\mathbb{R}^{N}\right)$ in $\dot{H}^{s}\left(\mathbb{R}^{N}\right)$ we may assume that $u_{n} \in C_{0}^{\infty}\left(\mathbb{R}^{N}\right)$. We can choose $y_{n} \in \mathbb{R}^{N}$ and $\lambda_{n}>0$ such that

$$
u_{n}^{y_{n}, \lambda_{n}}(\cdot):=\lambda_{n}^{\frac{N-2 s}{2}} u_{n}\left(\lambda_{n} \cdot+y_{n}\right) \in C_{0}^{\infty}(\Omega)
$$

Since

$$
\left\|u_{n}^{y_{n}, \lambda_{n}}\right\|_{X_{0}}=\left\|u_{n}\right\|_{\left.\dot{H}_{(\mathbb{R}}^{N}\right)}, \quad \int_{\Omega}\left|u_{n}^{y_{n}, \lambda_{n}}\right|^{p} d x=\int_{\mathbb{R}^{N}}\left|u_{n}\right|^{p} d x,
$$

we get $\gamma(\Omega) \leq \gamma\left(\mathbb{R}^{N}\right)$. Thus, $\gamma(\Omega)=\gamma\left(\mathbb{R}^{N}\right)$.

Assume by contradiction that $\Omega \neq \mathbb{R}^{N}$ and $u \in X_{0}$ is a minimizer for $\gamma(\Omega)$. Let $t>0$ such that $t|u| \in \mathcal{M}(\Omega)$. Then

$$
t=\left(\frac{\||u|\|_{X_{0}}^{2}}{\int_{\Omega}|u|^{p} d x}\right)^{\frac{1}{p-2}} \leq\left(\frac{\|u\|_{X_{0}}^{2}}{\int_{\Omega}|u|^{p} d x}\right)^{\frac{1}{p-2}}=1
$$

Consequently,

$$
\gamma(\Omega) \leq J(t|u|)=t^{p}\left(\frac{1}{2}-\frac{1}{p}\right) \int_{\Omega}|u|^{p} d x \leq \gamma(\Omega) .
$$


Thus, $t=1$ and $|u| \in \mathcal{M}(\Omega)$ is another minimizer for $\gamma(\Omega)$. For this reason we assume straight away that $u \geq 0$. Clearly, $u \in \mathbb{R}^{\mathcal{N}}$ is a minimizer for $J_{\infty}$. Therefore, we get that $J_{\infty}^{\prime}(u)=0$. So that $u$ is a solution of

$$
(-\Delta)^{s} u=u^{p} \quad \text { in } \mathbb{R}^{N}
$$

By the maximum principle (Proposition 2.2.8 in [33]), $u>0$ in $\mathbb{R}^{N}$. This is a contradiction.

Lemma 4.5 If $u \in \mathcal{N}^{-}$satisfies $I(u) \leq m^{+}+\frac{s}{N} S_{s}^{\frac{N}{2 s}}$, then $\int_{\Omega} a(x)\left(u^{+}\right)^{p} d x>0$.

Proof Let $u \in \mathcal{N}^{-}$with $I(u) \leq m^{+}+\frac{s}{N} S_{s}^{\frac{N}{2 s}}$. Then there exists unique $t(u)>0$ such that $t(u) u \in \mathcal{M}(\Omega)$. Assume by contradiction that $\int_{\Omega} a(x)\left(u^{+}\right)^{p} d x \leq 0$. By Lemmas 2.4 and 4.4,

$$
\begin{aligned}
I(u) & =\sup _{t \geq 0} I(t u) \geq I(t(u) u) \geq J(t(u) u)-\frac{1}{p} \int_{\Omega} a(x)\left(t(u) u^{+}\right)^{p} d x \\
& \geq \frac{s}{N} S_{s}^{\frac{N}{2 s}}-\frac{t^{p}(u)}{p} \int_{\Omega} a(x)\left(u^{+}\right)^{p} d x .
\end{aligned}
$$

Hence, by Lemma 3.1,

$$
\frac{t^{p}(u)}{p} \int_{\Omega} a(x)\left(u^{+}\right)^{p} d x \geq-m^{+}>0
$$

We get a contradiction.

\section{Existence of the other two solutions}

For $\mu>0$, we define

$$
\begin{aligned}
& I_{\mu}(u)=\frac{1}{2}\|u\|_{X_{0}}^{2}-\frac{\mu}{2_{s}^{*}} \int_{\Omega}|u|^{2_{s}^{*}} d x, \\
& \mathcal{N}_{\mu}=\left\{u \in X_{0} \backslash\{0\}:\left\langle I_{\mu}^{\prime}(u), u\right\rangle=0\right\} .
\end{aligned}
$$

Lemma 5.1 For each $u \in \mathcal{N}^{-}$, we have

(i) there exists unique $t_{\mu}(u)>0$ such that $t_{\mu}(u) u \in \mathcal{N}_{\mu}$, and

$$
\sup _{t \geq 0} I_{\mu}(t u)=I_{\mu}\left(t_{\mu}(u) u\right)=\frac{s}{N}\left(\frac{\|u\|_{X_{0}}^{2_{s}^{*}}}{\mu \int_{\Omega}|u|^{2_{s}^{*}} d x}\right)^{\frac{N-2 s}{2 s}}
$$

(ii) there exists unique $t(u)>0$ such that $t(u) u \in \mathcal{M}(\Omega)$, and for $c \in(0,1)$,

$$
J(t(u) u) \leq(1-c)^{-\frac{N}{2 s}}\left(I(u)+\frac{2-p}{2 p} c^{\frac{p}{p-2}}\left(\left|a^{+}\right|_{q} S_{s}^{-\frac{p}{2}}\right)^{\frac{2}{2-p}}\right)
$$

Proof (i) The proof is standard, and we omit it.

(ii) Let $\mu=(1-c)^{-1}$. Then, by Young's inequality,

$$
\int_{\Omega} a(x)\left(t_{\mu}(u) u^{+}\right)^{p} d x \leq\left|a^{+}\right|_{q} S_{s}^{-p / 2} t_{\mu}^{p}(u)\|u\|_{X_{0}}^{p}
$$




$$
\begin{aligned}
& \leq \frac{2-p}{2}\left(\left|a^{+}\right|_{q} S_{s}^{-p / 2} c^{-\frac{p}{2}}\right)^{\frac{2}{2-p}}+\frac{p}{2}\left(c^{\frac{p}{2}} t_{\mu}^{p}(u)\|u\|_{X_{0}}^{p}\right)^{\frac{2}{p}} \\
& =\frac{2-p}{2} c^{\frac{p}{p-2}}\left(\left|a^{+}\right|_{q} S_{s}^{-p / 2}\right)^{\frac{2}{2-p}}+\frac{p c}{2} t_{\mu}^{2}(u)\|u\|_{X_{0}}^{2} .
\end{aligned}
$$

By Lemmas 3.1 and 2.4, we have $I(u) \geq m^{-}>0$ and $I(u)=\sup _{t \geq 0} I(t u)$. By (i), we have

$$
\begin{aligned}
I(u) & =\sup _{t \geq 0} I(t u) \\
& \geq I\left(t_{\mu}(u) u\right) \\
& \geq \frac{1-c}{2}\left\|t_{\mu}(u) u\right\|_{X_{0}}^{2}-\frac{2-p}{2 p} c^{\frac{p}{p-2}}\left(\left|a^{+}\right|_{q} S_{s}^{-p / 2}\right)^{\frac{2}{2-p}}-\frac{1}{2_{s}^{*}} \int_{\Omega}\left(t_{\mu}(u) u^{+}\right)^{2_{s}^{*}} d x \\
& \geq(1-c) I_{\mu}\left(t_{\mu}(u) u\right)-\frac{2-p}{2 p} c^{\frac{p}{p-2}}\left(\left|a^{+}\right|_{q} S_{s}^{-p / 2}\right)^{\frac{2}{2-p}} \\
& =(1-c)^{\frac{N}{2 s}} \frac{\|u\|_{X_{0}}^{2_{s}^{*}}}{N}\left(\frac{N-2 s}{\int_{\Omega}|u|^{*} d x}\right)^{\frac{N-2 s}{2 s}}-\frac{2-p}{2 p} c^{\frac{p}{p-2}}\left(\left|a^{+}\right|_{q} S_{s}^{-p / 2}\right)^{\frac{2}{2-p}} \\
& =(1-c)^{\frac{N}{2 s}} J(t(u) u)-\frac{2-p}{2 p} c^{\frac{p}{p-2}}\left(\left|a^{+}\right|_{q} S_{s}^{-p / 2}\right)^{\frac{2}{2-p}} .
\end{aligned}
$$

Thus, we get (5.1).

Lemma 5.2 There exists $\delta_{0}>0$ such that, for $u \in \mathcal{M}(\Omega)$ with $J(u) \leq \frac{s}{N} S_{s}^{\frac{N}{2 s}}+\delta_{0}$, we have

$$
\int_{\mathbb{R}^{2 N}} \frac{x}{|x|}|u(x)-u(y)|^{2} K(x-y) d x d y \neq 0
$$

Proof Assume by contradiction that there exists a sequence $\left\{u_{n}\right\} \subset \mathcal{M}(\Omega)$ such that

$$
J\left(u_{n}\right)=\frac{s}{N} S_{s}^{\frac{N}{2 s}}+o(1) \quad \text { and } \quad \int_{\mathbb{R}^{2 N}} \frac{x}{|x|}\left|u_{n}(x)-u_{n}(y)\right|^{2} K(x-y) d x d y=0 .
$$

Without loss of generality, we can assume that $\left\{u_{n}\right\}$ is a $(P S)_{\gamma(\Omega)}$-sequence (for example, see Lemma 7 in [38]) for $J$. Since $J$ is coercive on $\mathcal{M}(\Omega)$, there exists a subsequence of $\left\{u_{n}\right\}$ (still denoted by $\left.\left\{u_{n}\right\}\right)$ and $u_{0} \in X_{0}$ such that $u_{n} \rightarrow u_{0}$ in $X_{0}$. Since $\Omega$ is a bounded domain, we have $u_{0} \equiv 0$. By Theorem 1.1 in [23] and Lemma 4.4, there exist $\ell$ nontrivial solutions $v^{1}, \ldots, v^{\ell} \in \dot{H}^{s}\left(\mathbb{R}^{N}\right)$ to

$$
(-\Delta)^{s} u=|u|^{2_{s}^{*}-2} u \text { in } \mathbb{R}^{N},
$$

or

$$
(-\Delta)^{s} u=|u|^{2_{s}^{*}-2} u \quad \text { in } \mathbb{R}_{+}^{N}, \quad u=0 \quad \text { in } \mathbb{R}^{N} \backslash \mathbb{R}_{+}^{N},
$$

where $\ell \in \mathbb{N}$, sequences of points $x_{n}^{1}, \ldots, x_{n}^{\ell} \subset \Omega$ and finitely many sequences of numbers $r_{n}^{1}, \ldots, r_{n}^{\ell} \subset(0,+\infty)$ converging to zero such that, up to a subsequence,

$$
u_{n}=\sum_{j=1}^{\ell}\left(r_{n}^{j}\right)^{\frac{2 s-N}{2}} v^{j}\left(\frac{x-x_{n}^{j}}{r_{n}^{j}}\right)+o(1) \quad \text { in } \dot{H}^{s}\left(\mathbb{R}^{N}\right),
$$


and

$$
J\left(u_{n}\right)=\sum_{j=1}^{\ell} J_{\infty}\left(v^{j}\right)+o(1)
$$

If $\ell>1$, then by (5.6) we have $J\left(u_{n}\right) \rightarrow \sum_{j=1}^{\ell} J_{\infty}\left(v^{j}\right)>\gamma(\Omega)$, which is a contradiction. Thus, by $(5.5)$,

$$
u_{n}=\left(r_{n}^{1}\right)^{\frac{2 s-N}{2}} v^{1}\left(\frac{x-x_{n}^{1}}{r_{n}^{1}}\right)+o(1) \text { in } \dot{H}^{s}\left(\mathbb{R}^{N}\right) .
$$

By (H1), $\left|x_{n}^{1}\right|$ is bounded from below. Hence, we may assume $\frac{x_{n}^{1}}{\left|x_{n}^{1}\right|} \rightarrow \mathbf{e}$ as $n \rightarrow \infty$, where $|\mathbf{e}|=1$. By Lebesgue's dominated convergence theorem, we have

$$
\begin{aligned}
0 & =\int_{\mathbb{R}^{2 N}} \frac{x}{|x|}\left|u_{n}(x)-u_{n}(y)\right|^{2} K(x-y) d x d y \\
& =\int_{\mathbb{R}^{2 N}} \frac{r_{n}^{1} \tilde{x}+x_{n}^{1}}{\left|r_{n}^{1} \tilde{x}+x_{n}^{1}\right|}\left|v^{1}(\tilde{x})-v^{1}(\tilde{y})\right|^{2} K(\tilde{x}-\tilde{y}) d \tilde{x} d \tilde{y}+o(1) \\
& =\mathbf{e} S_{s}^{\frac{N}{2 s}}+o(1)
\end{aligned}
$$

which is impossible.

Lemma 5.3 There exists $\sigma_{0} \in\left(0, \sigma_{2}\right)$ such that, for $\left|a^{+}\right|_{q} \in\left(0, \sigma_{0}\right)$, we have

$$
\int_{\mathbb{R}^{2 N}} \frac{x}{|x|}|u(x)-u(y)|^{2} K(x-y) d x d y \neq 0
$$

for all $u \in \mathcal{N}^{-}$with $I(u)<m^{+}+\frac{s}{N} S_{s}^{\frac{N}{2 s}}$

Proof For $u \in \mathcal{N}^{-}$with $I(u)<m^{+}+\frac{s}{N} S_{s}^{\frac{N}{2 s}}$, there exists $t(u)>0$ such that $t(u) u \in \mathcal{M}(\Omega)$. By Lemma 5.1(ii), for any $c \in(0,1)$, we have

$$
\begin{aligned}
J(t(u) u) & \leq(1-c)^{-\frac{N}{2 s}}\left(I(u)+\frac{2-p}{2 p} c^{\frac{p}{p-2}}\left(\left|a^{+}\right|_{q} S^{-\frac{p}{2}}\right)^{\frac{2}{2-p}}\right) \\
& <(1-c)^{-\frac{N}{2 s}}\left(\frac{s}{N} S_{s}^{\frac{N}{2 s}}+\frac{2-p}{2 p} c^{\frac{p}{p-2}}\left(\left|a^{+}\right|_{q} S_{s}^{-\frac{p}{2}}\right)^{\frac{2}{2-p}}\right),
\end{aligned}
$$

since $m^{+}<0$ by Lemma 3.1. Thus, there exists $\sigma_{0} \in\left(0, \sigma_{2}\right)$ such that, for $a \in C(\bar{\Omega})$ with $\left|a^{+}\right|_{q} \in\left(0, \sigma_{0}\right)$,

$$
J(t(u) u)<\frac{s}{N} S_{s}^{\frac{N}{2 s}}+\delta_{0}
$$

where $\delta_{0}$ is given in Lemma 5.2. Consequently, by Lemma 5.2,

$$
\int_{\mathbb{R}^{2 N}} \frac{x}{|x|}|t(u) u(x)-t(u) u(y)|^{2} K(x-y) d x d y \neq 0
$$

Hence, we complete the proof. 
Now, we use Lusternik and Schnirelmann's theory in order to obtain multiplicity results. The notion of category was introduced by Lusternik and Schnirelmann. It is a topological tool used in the estimate of the lower bounded of the number of critical points of a functional.

Definition 5.4 Let $\mathfrak{X}$ be a topological space. A closed subset $A$ of $\mathfrak{X}$ is contractible in $\mathfrak{X}$ if there exists $h \in C([0,1] \times A, \mathfrak{X})$ and $v \in \mathfrak{X}$ such that, for every $u \in A$,

$$
h(0, u)=u, \quad h(1, u)=v .
$$

Definition 5.5 The (L-S) category of $A$ with respect to $\mathfrak{X}$ (or simply the category of $A$ with respect to $\mathfrak{X})$, denoted by cat $\mathfrak{x}(A)$, is the least integer $k$ such that $A \subset A_{1} \cup \cdots \cup A_{k}$, with $A_{i}(i=1, \ldots, k)$ closed and contractible in $\mathfrak{X}$.

We set $\operatorname{cat}_{\mathfrak{X}}(\emptyset)=0$ and $\operatorname{cat}_{\mathfrak{X}}(A)=+\infty$ if there are no integers with the above property. We will use the notation $\operatorname{cat}(\mathfrak{X})$ for cat $\mathfrak{X}(\mathfrak{X})$. For fundamental properties of LusternikSchnirelmann category, we refer to Ambrosetti [2], Schwartz [27], and Chang [10].

Theorem 5.6 (Lusternik-Schnirelmann theorem) Let $M$ be a smooth Banach-Finsler manifold. Suppose that $f \in C^{1}(M, \mathbb{R})$ is a functional bounded from below, satisfying the $(P S)$ condition. Then $f$ has at least cat $(M)$ critical points.

We say $f$ satisfies the $(P S)$ condition if any sequence $\left\{u_{n}\right\} \subset M$, such that

$$
\left|f\left(u_{n}\right)\right| \leq \text { const. and } f^{\prime}\left(u_{n}\right) \rightarrow 0 \text {, }
$$

has a converging subsequence.

The following lemma is from [1].

Lemma 5.7 Let $\mathfrak{X}$ be a topological space. Suppose that there exist two continuous maps

$$
F: \mathbb{S}^{N-1} \rightarrow \mathfrak{X}, \quad G: \mathfrak{X} \rightarrow \mathbb{S}^{N-1}
$$

such that $G \circ F$ is homotopic to identity map of $\mathbb{S}^{N-1}$, that is, there exists $\xi \in C([0,1] \times$ $\left.\mathbb{S}^{N-1}, \mathbb{S}^{N-1}\right)$ such that

$$
\begin{aligned}
& \xi(0, x)=(G \circ F)(x) \quad \text { for all } x \in \mathbb{S}^{N-1}, \\
& \xi(1, x)=x \text { for all } x \in \mathbb{S}^{N-1} .
\end{aligned}
$$

Then

$$
\operatorname{cat}(\mathfrak{X}) \geq 2 \text {. }
$$

For $\varepsilon<\varepsilon_{0}\left(\varepsilon_{0}\right.$ is defined in Lemma 4.2), we define a map $\Phi: \mathbb{S}^{N-1} \rightarrow X_{0}$ by

$$
\Phi(\mathbf{e})=\tilde{u}_{1}+s_{0} t_{0} u_{\varepsilon, \mathbf{e}} \quad \text { for all } \mathbf{e} \in \mathbb{S}^{N-1},
$$

where $s_{0}, t_{0}$ are given in Lemma 4.3. 
Lemma $5.8 \Phi\left(\mathbb{S}^{N-1}\right)$ is compact.

Proof Let $\left\{\mathbf{e}_{n}\right\} \subset \mathbb{S}^{N-1}$ be a sequence such that $\mathbf{e}_{n} \rightarrow \mathbf{e}_{0}$ as $n \rightarrow \infty$. Using a similar argument as that in the proof of Lemma 4.1 and Lebesgue's dominated convergence theorem, we obtain $\left\|u_{\varepsilon, \mathbf{e}_{n}}\right\|_{X_{0}} \rightarrow\left\|u_{\varepsilon, \mathbf{e}_{0}}\right\|_{X_{0}}$ as $n \rightarrow \infty$. Since $X_{0}$ is a Hilbert space and $u_{\varepsilon, \mathbf{e}_{n}} \rightarrow u_{\varepsilon, \mathbf{e}_{0}}$, we get $\left\|u_{\varepsilon, \mathbf{e}_{n}}-u_{\varepsilon, \mathbf{e}_{0}}\right\|_{X_{0}} \rightarrow 0$. Consequently, $\Phi\left(\mathbf{e}_{n}\right) \rightarrow \Phi\left(\mathbf{e}_{0}\right)$.

For $c \in \mathbb{R}$, we define

$$
I^{c}:=\left\{u \in \mathcal{N}^{-}: I(u) \leq c\right\}
$$

Lemma 5.9 There exists $d_{\varepsilon} \in\left(0, m^{+}+\frac{s}{N} S_{s}^{\frac{N}{2 s}}\right)$ such that $\Phi\left(\mathbb{S}^{N-1}\right) \subset I^{d_{\varepsilon}}$ for each $\varepsilon \in\left(0, \varepsilon_{0}\right)$.

Proof By Lemmas 4.2 and 4.3(iii), for each $\varepsilon \in\left(0, \varepsilon_{0}\right)$, we have $\tilde{u}_{1}+s_{0} t_{0} u_{\varepsilon, \mathbf{e}} \in \mathcal{N}^{-}$and

$$
\sup _{t \geq 0} I\left(\tilde{u}_{1}+t u_{\varepsilon, \mathbf{e}}\right)<m^{+}+\frac{s}{N} S_{s}^{\frac{N}{2 s}}
$$

uniformly in $\mathbf{e} \in \mathbb{S}^{N-1}$. Since $\Phi\left(\mathbb{S}^{N-1}\right)$ is compact by Lemma 5.8 , there exists $d_{\varepsilon} \in\left(0, m^{+}+\right.$ $\left.\frac{S}{N} S_{s}^{\frac{N}{2 S}}\right)$ such that $\Phi\left(\mathbb{S}^{N-1}\right) \subset I^{d_{\varepsilon}}$.

Set $\beta=m^{+}+\frac{s}{N} S_{s}^{\frac{N}{2 s}}$ and define $\Psi: I^{\beta} \rightarrow \mathbb{S}^{N-1}$ by

$$
\Psi(u)=\frac{\int_{\mathbb{R}^{2 N}} \frac{x}{|x|}|u(x)-u(y)|^{2} K(x-y) d x d y}{\left|\int_{\mathbb{R}^{2 N}} \frac{x}{|x|}\right| u(x)-\left.u(y)\right|^{2} K(x-y) d x d y \mid} .
$$

By Lemma 5.3, $\Psi$ is well-defined. Let

$$
\Sigma=\left\{u \in X_{0} \backslash\{0\}: \int_{\mathbb{R}^{2 N}} \frac{x}{|x|}|u(x)-u(y)|^{2} K(x-y) d x d y \neq 0\right\} .
$$

We define $\widetilde{\Psi}: \Sigma \rightarrow \mathbb{S}^{N-1}$ by

$$
\widetilde{\Psi}(u)=\frac{\int_{\mathbb{R}^{2 N}} \frac{x}{|x|}|u(x)-u(y)|^{2} K(x-y) d x d y}{\left|\int_{\mathbb{R}^{2 N}} \frac{x}{|x|}\right| u(x)-\left.u(y)\right|^{2} K(x-y) d x d y \mid} .
$$

Clearly, $\widetilde{\Psi}$ is an extension of $\Psi$.

Lemma 5.10 $u_{\varepsilon, \mathbf{e}} \in \Sigma$ for all $\mathbf{e} \in \mathbb{S}^{N-1}$ and for $\varepsilon$ small enough.

Proof For every $u_{\varepsilon, \mathbf{e}}$, one sees immediately that there exists $t(\varepsilon, \mathbf{e})>0$ such that $t(\varepsilon, \mathbf{e}) u_{\varepsilon, \mathbf{e}} \in$ $\mathcal{M}(\Omega)$. Indeed, $t(\varepsilon, \mathbf{e}) u_{\varepsilon, \mathbf{e}} \in \mathcal{M}(\Omega)$ is equivalent to

$$
\left\|t(\varepsilon, \mathbf{e}) u_{\varepsilon, \mathbf{e}}\right\|_{X_{0}}^{2}=\int_{\Omega}\left|t(\varepsilon, \mathbf{e}) u_{\varepsilon, \mathbf{e}}\right|^{2_{s}^{*}} d x,
$$

which is solved by

$$
t(\varepsilon, \mathbf{e})=\left(\frac{\left\|u_{\varepsilon, \mathbf{e}}\right\|_{X_{0}}^{2}}{\int_{\Omega}\left|u_{\varepsilon, \mathbf{e}}\right|^{2 *} d x}\right)^{1 /\left(2_{s}^{*}-2\right)} .
$$


By Lemma 4.1, we have

$$
\lim _{\varepsilon \rightarrow 0} t(\varepsilon, \mathbf{e})=1
$$

uniformly in $\mathbf{e} \in \mathbb{S}^{N-1}$. Thus,

$$
\lim _{\varepsilon \rightarrow 0} J\left(t(\varepsilon, \mathbf{e}) u_{\varepsilon, \mathbf{e}}\right)=\frac{s}{N} S_{s}^{\frac{N}{2 s}}
$$

uniformly in $\mathbf{e} \in \mathbb{S}^{N-1}$. By Lemma 5.2 , we get $t(\varepsilon, \mathbf{e}) u_{\varepsilon, \mathbf{e}} \in \Sigma$ for $\varepsilon>0$ small enough. Consequently, $u_{\varepsilon, \mathbf{e}} \in \Sigma$.

Lemma 5.11 $\Psi \circ \Phi: \mathbb{S}^{N-1} \rightarrow \mathbb{S}^{N-1}$ is homotopic to the identity.

Proof By Lemma 5.10, there exists $\varepsilon^{*} \in\left(0, \varepsilon_{0}\right)$ such that, for $\varepsilon \in\left(0, \varepsilon^{*}\right), u_{\varepsilon, \mathbf{e}} \in \Sigma$ and $u_{2(1-\theta) \varepsilon, \mathbf{e}} \in \Sigma$ for all $\mathbf{e} \in \mathbb{S}^{N-1}$ and $\theta \in\left[\frac{1}{2}, 1\right)$. Let $\gamma:\left[s_{1}, s_{2}\right] \rightarrow \mathbb{S}^{N-1}$ be a regular geodesic between $\widetilde{\Psi}\left(u_{\varepsilon, \mathbf{e}}\right)$ and $\widetilde{\Psi}(\Phi(\mathbf{e}))$ such that

$$
\gamma\left(s_{1}\right)=\tilde{\Psi}\left(u_{\varepsilon, \mathbf{e}}\right), \quad \gamma\left(s_{2}\right)=\tilde{\Psi}(\Phi(\mathbf{e})) .
$$

Define $\xi:[0,1] \times \mathbb{S}^{N-1} \rightarrow \mathbb{S}^{N-1}$ by

$$
\xi(\theta, \mathbf{e})= \begin{cases}\left.\gamma\left(2 \theta s_{1}+(1-2 \theta) s_{2}\right)\right) & \text { for } \theta \in\left[0, \frac{1}{2}\right), \\ \widetilde{\Psi}\left(u_{2(1-\theta) \varepsilon, \mathbf{e}}\right) & \text { for } \theta \in\left[\frac{1}{2}, 1\right), \\ \mathbf{e} & \text { for } \theta=1 .\end{cases}
$$

Set $\tilde{x}=(x-\tilde{\rho} \mathbf{e}) /(2(1-\theta) \varepsilon), \tilde{y}=(y-\tilde{\rho} \mathbf{e}) /(2(1-\theta) \varepsilon)$. Then

$$
\begin{aligned}
& \int_{\mathbb{R}^{2 N}} \frac{x}{|x|}\left|u_{2(1-\theta) \varepsilon, \mathbf{e}}(x)-u_{2(1-\theta) \varepsilon, \mathbf{e}}(y)\right|^{2} K(x-y) d x d y \\
& \quad=\int_{\mathbb{R}^{2 N}} \frac{\tilde{\rho} \mathbf{e}+2(1-\theta) \varepsilon \tilde{x}}{|\tilde{\rho} \mathbf{e}+2(1-\theta) \varepsilon \tilde{x}|} \eta(\tilde{\rho} \mathbf{e}+2(1-\theta) \varepsilon \tilde{x})\left|U_{1}(\tilde{x})-U_{1}(\tilde{y})\right|^{2} K(\tilde{x}-\tilde{y}) d \tilde{x} d \tilde{y} \\
& \quad \rightarrow S_{S}^{\frac{N}{2 s}} \mathbf{e}
\end{aligned}
$$

as $\theta \rightarrow 1^{-}$by (4.4) and Lebesgue's dominated convergence theorem. Consequently,

$$
\lim _{\theta \rightarrow 1^{-}} \xi(\theta, \mathbf{e})=\mathbf{e} .
$$

Clearly, $\xi(\theta, \mathbf{e}) \rightarrow \gamma(s-1)=\widetilde{\Psi}\left(u_{\varepsilon, \mathbf{e}}\right)$ as $\theta \rightarrow \frac{1}{2}^{-}$. Thus, $\xi \in C\left([0,1] \times \mathbb{S}^{N-1}, \mathbb{S}^{N-1}\right)$, and

$$
\begin{aligned}
& \xi(0, \mathbf{e})=\widetilde{\Psi}(\Phi(\mathbf{e})), \\
& \xi(1, \mathbf{e})=\mathbf{e},
\end{aligned}
$$

for all $\mathbf{e} \in \mathbb{S}^{N-1}$. 
Proof of Theorem 1.1 By Lemmas 5.7, 5.9, and 5.11, there exists $d_{\varepsilon} \in\left(0, m^{+}+\frac{s}{N} S_{s}^{\frac{N}{2 s}}\right)$ such that

$$
\operatorname{cat}\left(I^{d_{\varepsilon}}\right) \geq 2
$$

By Lemma 3.2 and Theorem 5.6, $I$ has at least two critical points $\tilde{u}_{2}$ and $\tilde{u}_{3}$ in $\left\{u \in \mathcal{N}^{-}\right.$: $\left.I(u)<m^{+}+\frac{s}{N} S_{s}^{\frac{N}{2 s}}\right\}$. By the maximum principle (Proposition 2.2.8 in [33]), $\tilde{u}_{2}$ and $\tilde{u}_{3}$ are strictly positive in $\Omega$. By Theorem 3.3, we get three positive solutions $\tilde{u}_{i}(i=1,2,3)$ of (1.1). By (2.5) and Lemma 4.5, we have $\int_{\Omega} a(x) \tilde{u}_{i}^{p}>0, i=1,2,3$.

\section{Acknowledgements}

Not applicable.

\section{Funding}

This work is partially supported by NNSFC (No. 11871315).

\section{Availability of data and materials}

Not applicable.

\section{Competing interests}

The author declares that he/she has no competing interests.

\section{Authors' contributions}

YZ contributed the central idea and wrote the initial draft of the paper. The other authors contributed to refining the ideas, carrying out additional analyses, and finalizing this paper. All authors read and approved the final manuscript.

\section{Authors' information}

Not applicable.

\section{Publisher's Note}

Springer Nature remains neutral with regard to jurisdictional claims in published maps and institutional affiliations.

Received: 11 February 2019 Accepted: 9 April 2019 Published online: 24 April 2019

\section{References}

1. Adachi, S., Tanaka, K.: Four positive solutions for the semilinear elliptic equation: $-\Delta u+u=a(x) u^{p}+f(x)$ in $\mathbb{R}^{N}$. Calc. Var. 11, 63-95 (2000)

2. Ambrosetti, A.: Critical Points and Nonlinear Variational Problems. Mémoires de la S. M. F. $2^{e}$ série, vol. 49 (1992)

3. Atangana, A., Baleanu, D.: New fractional derivatives with nonlocal and non-singular kernel: theory and application to heat transfer model. Therm. Sci. 20, 763-769 (2009)

4. Bahri, A., Coron, J.M.: On a nonlinear elliptic equation involving the critical Sobolev exponent: the effect of the topology of the domain. Commun. Pure Appl. Math. 41, 253-294 (1988)

5. Bahri, A., Li, Y.Y.: On the min-max procedure for the existence of a positive solution for certain scalar field equations in $\mathbb{R}^{N}$. Rev. Mat. Iberoam. 6, 1-15 (1990)

6. Barriosa, B., Colorado, E., Servadeid, R., Soria, F.: A critical fractional equation with concave-convex power nonlinearities. Ann. Inst. Henri Poincaré, Anal. Non Linéaire 32, 875-900 (2015)

7. Ben Omrane, I., Gala, S., Kim, J.-M., Ragusa, M.A.: Logarithmically improved blow-up criterion for smooth solutions to the Leray- $\alpha$-magnetohydrodynamic equations. Arch. Math. 55, 55-68 (2019)

8. Brézis, H., Lieb, E.H.: A relation between pointwise convergence of functions and convergence of functionals. Proc. Am. Math. Soc. 88, 483-490 (1983)

9. Brown, K.J., Zhang, Y.: The Nehari manifold for a semilinear elliptic equation with a sign-changing weight function. J. Differ. Equ. 193, 481-499 (2003)

10. Chang, K.-C.: Methods in Nonlinear Analysis. Springer, Berlin (2005)

11. Cont, R., Tankov, P.: Financial Modeling with Jump Processes. Chapman \& Hall/CRC Financ. Math. Ser. Chapman and Hall/CRC, Boca Raton (2004)

12. Coron, J.M.: Topologie et cas limite des injections de Sobolev. C. R. Acad. Sci. Paris, Ser. I 299, 209-212 (1984)

13. Ekeland, I.: On the variational principle. J. Math. Anal. Appl. 17, 324-353 (1974)

14. Feng, M., Li, P., Sun, S.: Symmetric positive solutions for fourth-order $n$-dimensional $m$-Laplace systems. Bound. Value Probl. 2018, 63 (2018)

15. Guariglia, E., Silvestrov, S.: A functional equation for the Riemann zeta fractional derivative. AIP Conf. Proc. 1798 020063 (2017)

16. He, H., Yang, J.: Positive solutions for critical inhomogeneous elliptic problems in non-contractible domains. Nonlinear Anal. 70, 952-973 (2009)

17. Li, C., Dao, X., Guo, P.: Fractional derivatives in complex planes. Nonlinear Anal. 71, 1857-1869 (2009) 
18. Li, G., Yan, S., Yang, J.: An elliptic problem with critical growth in domains with shrinking holes. J. Differ. Equ. 198, 275-300 (2004)

19. Li, X., Pang, L., Zhang, Y.: Existence of multiple positive solutions for fractional Laplace problems with critical growth. Preprint

20. Liu, Z., Tan, J.: Nonlocal elliptic hemivariational inequalities. Electron. J. Qual. Theory Differ. Equ. 2017, 66 (2017)

21. Majda, A., Tabak, E.: A two-dimensional model for quasigeostrophic flow: comparison with the two-dimensional Euler flow. Nonlinear Phenomena in Ocean Dynamics, Los Alamos, NM, 1995, Physica D 98, 515-522 (1996)

22. Nezza, E.D., Palatucci, G., Valdinoci, E.: Hitchhikers guide to the fractional Sobolev spaces. Bull. Sci. Math. 136, 521-573 (2012)

23. Palatucci, G., Pisante, A.: A global compactness type result for Palais-Smale sequences in fractional spaces. Nonlinear Anal. 117, 1-7 (2015)

24. Ragusa, M.A.: Commutators of fractional integral operators in vanishing-Morrey spaces. J. Glob. Optim. 40, 361-368 (2008)

25. Ragusa, M.A., Tachikawa, A.: On continuity of minimizers for certain quadratic growth functionals. J. Math. Soc. Jpn. $57,691-700(2005)$

26. Ros-Oton, X., Serra, J.: The Dirichlet problem for the fractional Laplacian: regularity up to the boundary. J. Math. Pures Appl. 101, 275-302 (2014)

27. Schwartz, J.T.: Nonlinear Functional Analysis. Gordon \& Breach, New York (1969)

28. Secchi, S., Shioji, N., Squassina, M.: Coron problem for fractional equations. Differ. Integral Equ. 28, 103-118 (2015)

29. Servadei, R., Valdinoci, E.: Lewy-Stampacchia type estimates for variational inequalities driven by (non)local operators. Rev. Mat. Iberoam. 29, 1091-1126 (2013)

30. Servadei, R., Valdinoci, E.: Variational methods for the non-local operators of elliptic type. Discrete Contin. Dyn. Syst. 33, 2105-2137 (2013)

31. Servadei, R., Valdinoci, E.: Weak and viscosity solutions of the fractional Laplace equation. Publ. Mat. 58, 133-154 (2014)

32. Servadei, R., Valdinoci, E.: The Brezis-Nirenberg result for the fractional Laplacian. Trans. Am. Math. Soc. 367, 67-102 (2015)

33. Silvestre, L.: Regularity of the obstacle problem for a fractional power of the Laplace operator. Commun. Pure Appl. Math. 60, 67-112 (2007)

34. Tarantello, G.: On nonhomogeneous elliptic equations involving critical Sobolev exponent. Ann. Inst. Henri Poincaré, Anal. Non Linéaire 9, 281-304 (1992)

35. Valdinoci, E.: From the long jump random walk to the fractional Laplacian. Bol. Soc. Esp. Mat. Apl. 49, 33-44 (2009)

36. Vlahos, L., Isliker, H., Kominis, Y., Hizonidis, K.: Normal and anomalous diffusion: a tutorial. In: Bountis, T. (ed.) Order and Chaos, vol. 10. Patras University Press (2008)

37. Wan, Y., Yang, J.: The existence of multiple solutions of semilinear elliptic involving Sobolev critical exponent. Nonlinear Anal. 68, 2569-2593 (2008)

38. Wang, H., Wu, T.: Symmetry breaking in a bounded symmetry domain. Nonlinear Differ. Equ. Appl. 11, 361-377 (2004)

39. $\mathrm{Wu}, \mathrm{T}$.: Three positive solutions for Dirichlet problems involving critical Sobolev exponent and sign-changing weight. J. Differ. Equ. 249, 1549-1578 (2010)

40. Zhu, X:: A perturbation result on positive entire solutions of a semilinear elliptic equation. J. Differ. Equ. 92, 163-178 (1991)

\section{Submit your manuscript to a SpringerOpen ${ }^{\circ}$ journal and benefit from:}

- Convenient online submission

- Rigorous peer review

- Open access: articles freely available online

- High visibility within the field

- Retaining the copyright to your article

Submit your next manuscript at $\gg$ springeropen.com 